\title{
Suficiencia de Capital y Riesgo de Crédito en Carteras de Préstamos \\ Bancarios
}

Un modelo de impago que relaciona la suficiencia de capital con el riesgo de crédito, mide el riesgo de concentración, y determina límites individuales para los créditos.

\author{
Javier Márquez Diez-Canedo ${ }^{1}$ \\ Abril 2002
}

Documento de Investigación No. 2002-04

Dirección General de Análisis del Sistema Financiero

Banco de México

\footnotetext{
${ }^{1}$ El autor desea agradecer la colaboración de Maribell Rojas Garduño en la construcción de los ejemplos presentados, y muy especialmente a Calixto López Castañón, por su importante contribución en las pruebas de los teoremas 6.1 y 6.2 del apéndice, la elaboración de los resultados del último ejemplo y un sin número de discusiones que condujeron a mejorar considerablemente la consistencia del modelo. Otra persona que contribuyó significativamente a redondear algunos aspectos del modelo, fue José Luis Farah del ITAM. Las opiniones expresadas en este documento son responsabilidad exclusiva del autor y no necesariamente corresponden a las del Banco de México.
} 


\title{
Suficiencia de Capital y Riesgo de Crédito en Carteras de Préstamos Bancarios: \\ Un modelo de impago que relaciona la suficiencia de capital con el riesgo de crédito, mide el riesgo de concentración, y determina límites individuales para los créditos.
}

\author{
Javier Márquez Diez-Canedo \\ Banco de México
}

Abril 2002

\section{Resumen}

La búsqueda de una regla explícita que relacione el riesgo de crédito con la suficiencia de capital, es uno de los temas más importantes tanto para los reguladores como para los administradores de riesgo. Las metodologías actuales de riesgo de crédito dependen sobremanera de métodos numéricos para obtener la distribución de pérdidas de una cartera; debido a esto, la determinación de la suficiencia de capital y de límites individuales para los créditos, es un proceso empírico que requiere de un gran sustento computacional. La obtención de una medida de concentración y su contribución al riesgo en carteras de créditos así como la identificación de segmentos que exhiban concentración excesiva, son problemas que hasta el momento no se han podido resolver a pesar de su gran importancia.

Con base en las probabilidades de impago de los créditos y de sus respectivas covarianzas, se desarrolló un modelo que obtiene una forma funcional de la distribución de pérdidas, suponiendo que dicha distribución puede caracterizarse por dos parámetros. La representación del Valor en Riesgo (VaR) en términos de la media y la varianza, conduce a una cota inferior para el cociente de capitalización bancaria y la desigualdad resultante establece condiciones para que un banco cuente con suficiencia de capital. El índice de "Herfindahl-Hirschman" surge como una medida de concentración, que cuantifica de manera precisa la contribución de la concentración al riesgo de crédito total de una cartera. Se obtienen dos propiedades nuevas de este índice, que relacionan los límites individuales de los créditos con la concentración existente en los distintos segmentos en los que se pueda descomponer la cartera, de tal manera que se garantice la suficiencia de capital. Los ejercicios numéricos realizados hasta la fecha en carteras de créditos reales, arrojan resultados comparables con aquellos obtenidos usando otras metodologías, con una reducción considerable en esfuerzo computacional. A lo largo de este artículo, se discuten las implicaciones de esto para la administración de riesgos y la regulación.

Palabras clave: Suficiencia de capital, riesgo de concentración crediticia, índice de concentración Herfindahl-Hirschman, límite de crédito individual, valor en riesgo, modelos de impago. 


\section{INTRODUCCIÓN².}

Actualmente, en los paradigmas más aceptados para medir riesgo de crédito se pueden distinguir dos enfoques, a saber: el de "marcar a mercado" y el de "modelos de impago". Las diferencias entre ambos estriban, en primer término, en la forma en que se consideran las pérdidas. Mientras que en los modelos de impago un deudor sólo puede estar en uno de dos estados, es decir pago o impago, las pérdidas que resulten sólo se deben a los incumplimientos del deudor. Los modelos de "marcar a mercado" en contraste, consideran además las pérdidas resultantes del cambio en el valor de los créditos, debido a la migración de calidad de los mismos. Sin embargo, las mayores diferencias entre ambos paradigmas, se deben a la forma en que conjugan los diferentes elementos del riesgo de crédito para obtener la distribución de probabilidades de las pérdidas. Por ejemplo ${ }^{3}$, en CreditMetrics ${ }^{\mathrm{TM}}$ que es un modelo de "marcar a mercado", el componente principal es la matriz de transición que está relacionada con un sistema de calificación, y que provee el mecanismo probabilístico que modela la migración de la calidad de los créditos. Esto determina las pérdidas resultantes de los incumplimientos del deudor, y los cambios en el valor de mercado de los créditos de la cartera, a través de un proceso de simulación Montecarlo que toma en cuenta explícitamente la matriz de covarianzas de migración, para finalmente obtener la distribución de pérdidas de la cartera. La matriz de transición, los cambios en el valor y las pérdidas debidas al incumplimiento de los créditos, así como las covarianzas de migración e incumplimiento se estiman a partir de datos estadísticos e información de mercado. En contraste, el proceso de simulación depende en gran medida de un supuesto de normalidad que permite establecer una relación entre la calidad del crédito y el valor de los activos de las empresas deudoras y aplicar el modelo de Merton ${ }^{4}$, así como determinar el comportamiento de migración conjunta de los créditos de la cartera.

La metodología $\mathbf{K M V} \mathbf{V}^{5}$ también se basa en el modelo de Merton. Define una "distancia al impago", que es la diferencia entre el valor de los activos de las empresas y un cierto umbral, de manera que si esta cantidad es negativa, la compañía estaría en bancarrota y no podría cumplir con sus obligaciones. Para propósitos de estandarización, esta "distancia al impago" se mide como un múltiplo de la desviación estándar del valor de los activos de las empresas. KMV ha acumulado una gran base de datos, que usa para estimar probabilidades de impago y correlaciones, así como las distribuciones de pérdidas debidas al incumplimiento del deudor y a las migraciones de calidad de

\footnotetext{
2 Un análisis detallado de las diferentes aproximaciones se presenta en M. Crouhy et al., JBF (24) 2000.

3 CreditMetrics ${ }^{\text {TM }}$ es un subproducto del grupo de desarrollo de los sistemas de Administración de Riesgos de J.P. Morgan.

4 Véase Merton 1974.

5 Esta metodología es propiedad de la corporación KMV.
} 
los créditos. Para una compañía en particular, esta probabilidad se aproxima por las "frecuencias esperadas de impago"; es decir, el cociente entre el número de compañías que están a la misma "distancia de impago" que realmente incumplieron, y el número total de compañías que están a la misma "distancia al impago" contenidas en la base de datos. Aunque también es un método de marcar a mercado, difiere significativamente de CreditMetrics ${ }^{\text {TM }}$ ya que depende de las "FEI" de cada deudor, en lugar de depender de las probabilidades de transición estimadas de los datos históricos, ya sea internos de cada banco o producidas por las agencias calificadoras. Existen también diferencias considerables en los supuestos y en las formas funcionales utilizadas.

CreditRisk $^{+6}$ es un modelo de impago en donde la piedra angular de la metodología, es el conjunto de probabilidades individuales de impago de los créditos en la cartera, y el supuesto de que las probabilidades de impago siempre son pequeñas, de manera que el número de incumplimientos en la cartera se puede aproximar adecuadamente por una distribución de probabilidad Poisson. En su versión más general, donde las probabilidades de impago pueden cambiar en el tiempo, se supone además que estas probabilidades están completamente explicadas por una suma ponderada de " $\boldsymbol{K}$ factores de riesgo" cada uno de ellos distribuido de acuerdo a una distribución Gamma independiente. Los ponderadores de los factores de riesgo difieren dependiendo de la calificación individual del deudor $\mathrm{y}$, condicionados a estos factores, se supone que los incumplimientos de los deudores individuales se comportan como ensayos Bernoulli independientes. En este caso general, la correlación de impago está implícita en los patrones de covariación entre los factores de riesgo, y el supuesto Poisson conduce a una distribución Binomial Negativa para el número de incumplimientos. Una vez obtenida la distribución del número de incumplimientos en la cartera, procediendo con un enfoque actuarial, se selecciona una unidad de pérdida y dadas las tasas de recuperación de los créditos individuales, éstos se agrupan en bandas donde la pérdida es la misma para cada crédito que cae en incumplimiento. Con esto, se obtiene la función generadora de probabilidad de las pérdidas. Finalmente, se recurre a un procedimiento numérico de recursión para obtener la distribución de probabilidad de pérdidas. Este procedimiento involucra el cálculo de los coeficientes de dos polinomios cuyo orden es igual al producto del número de bandas y el número de factores de riesgo en un caso, y el producto del número de bandas menos uno y el número de factores de riesgo en el otro caso. Este cálculo puede implicar un esfuerzo computacional importante, si la cartera es grande y la unidad de pérdida escogida es pequeña.

\footnotetext{
${ }^{6}$ CreditRisk $^{+}$es comercializado por Productos Financieros de Crédito Suizos.
} 
Otra metodología popular de impago es Credit Portfolio View ${ }^{7}$, que es un modelo discreto de periodos múltiples. Además del hecho de que esta metodología se concibe en un principio como un modelo dinámico, su atracción principal radica en que obtiene las probabilidades de impago como funciones logit de índices de variables macroeconómicas, que de alguna manera representan el desempeño de la economía. La cartera se segmenta de acuerdo a la localización geográfica y a la actividad económica de los deudores, y se supone que los índices para cada segmento son funciones lineales de las variables macroeconómicas asociadas a cada segmento. Este modelo supone que cada variable macroeconómica sigue un proceso autoregresivo de segundo orden, y debido a las correlaciones en los errores de los modelos lineales para los índices y de las expresiones autoregresivas de las variables macroeconómicas subyacentes, los parámetros de ambas se estiman simultáneamente de un sistema de ecuaciones. Como CreditMetrics ${ }^{\mathrm{TM}}$, Credit Portfolio View también recurre a la simulación de migraciones de calidad de los créditos a través de matrices de transición para obtener la distribución de las pérdidas. En este proceso, las matrices de transición incondicionales generadas por las agencias calificadoras se ajustan al estado de la economía de cada segmento, reduciendo o incrementando las probabilidades incondicionales de transición de acuerdo al cociente entre la probabilidad de impago simulada y la tasa promedio histórica incondicional de impago del segmento.

En una contribución reciente, Kreinin y Rosen (1999) proponen un modelo dinámico de etapas múltiples donde las probabilidades de impago se hacen depender de la evolución conjunta de los factores de riesgo de mercado y de crédito. Esto facilita la conjunción del riesgo de mercado y de crédito, con la virtud adicional de que el modelo puede cuantificar mejor la exposición de los segmentos a la contraparte, así como la posición en derivados de la cartera. Otra mejora en estos modelos recientes de riesgo de crédito es la posibilidad de modelar las exposiciones estocásticas mediante simulación, y la extensión del modelo de impago de Merton a etapas múltiples. La distribución de pérdidas se obtiene por simulación bajo la idea de "Marcar a Futuro" de las exposiciones de la contraparte y en el contexto de la probabilidad condicional de impago, alcanzando una gran eficiencia computacional.

Todas las metodologías mencionadas han contribuido al entendimiento de las ideas principales de los modelos de riesgo de crédito y ahora se acepta que todos los modelos están convergiendo a producir resultados comparables. Los resultados de Crouhy et. al. (2000), Finger (1998) y Gordy (2000) indican que, bajo ciertas equivalencias paramétricas, las metodologías principales como CreditMetrics $^{\text {TM }}$ y CreditRisk $^{+}$pueden "mapearse" una en la otra. Es importante notar que el

\footnotetext{
${ }^{7}$ Este producto lo ofrece McKinsey, la compañía consultora. La referencia clásica es Wilson (I) \& (II) 1997.
} 
énfasis de todas estas metodologías es producir una distribución de pérdidas, que se apega lo más posible a la realidad. Aunque difícilmente se puede ir en contra de este principio, el esfuerzo computacional requerido puede resultar impráctico para ciertos usuarios como los reguladores, quienes tienen que supervisar todo el sistema financiero, y no sólo un banco en particular. Además, el desarrollo de herramientas de gestión administrativa, tales como la obtención de reglas simples para establecer suficiencia de capital, la identificación de segmentos que presentan las mayores concentraciones de riesgo y el establecimiento de límites individuales para los créditos, que están directamente relacionados con el perfil de riesgo de la cartera, no se pueden obtener directamente.

El modelo que aquí se presenta, supone que están dadas las probabilidades de incumplimiento de los créditos y sus covarianzas. Con estos elementos, se desarrolla un modelo de impago que obtiene una forma funcional de la distribución de pérdidas, suponiendo que puede caracterizarse por dos parámetros: la media y la varianza. Dada una distribución de pérdidas con una media y varianza específicas, no necesariamente Normal, es posible obtener el Valor en Riesgo (VaR) de la cartera como la pérdida esperada más un cierto múltiplo de la desviación estándar de las pérdidas. Esto conduce a una cota inferior de la razón de capitalización del banco y la desigualdad que resulte establece la suficiencia de capital. Del desarrollo del modelo emerge de manera natural el índice de "Herfindahl-Hirschman" como medida de concentración, proporcionando una cuantificación precisa de la manera en que la concentración contribuye al riesgo de crédito de la cartera. Dos propiedades nuevas que se obtienen de este índice, relacionan los límites individuales de los créditos con la concentración existente en los distintos segmentos en los que se dividió la cartera, de tal manera que se garantice la suficiencia de capital. Los ejercicios numéricos realizados hasta la fecha en carteras reales, muestran resultados comparables con aquellos obtenidos usando otras metodologías. Además, se observa que las distribuciones obtenidas aproximan aquéllas que se obtienen con las metodologías más estándar, pero con una reducción considerable en esfuerzo computacional. Dado que la medición de concentración es el corazón del modelo, se comenzará con la discusión de este tema.

\section{EI Tema de la Concentración.}

La concentración de los créditos ha sido identificada como una fuente importante de riesgo para los bancos desde hace mucho tiempo. A juzgar por la literatura actual relativa al riesgo crediticio, al menos para el tema de concentración, el establecimiento de paradigmas generalmente aceptados no 
se ha materializado, a pesar de la importancia del problema ${ }^{8}$. Los acercamientos más formales, basados en la teoría de cartera ${ }^{9}$, se han enfocado principalmente a la diversificación óptima de carteras de instrumentos de renta fija, para los cuales se puede obtener información compatible con los modelos tradicionales de Markowitz (1959). Altman y Saunders (1998) presentan un enfoque interesante, que trata de evitar los principales problemas asociados con los datos y su análisis, al mismo tiempo que explota las virtudes de la teoría moderna de cartera. Sin embargo, se debe recalcar que los estudios basados en la teoría tradicional de cartera tratan el problema de concentración indirectamente, ya que su principal preocupación es la diversificación de los activos a través del conocido esquema de media-varianza, pero sin proporcionar una medida de concentración clara ni una relación explícita con el riesgo de la cartera. Kealhoffer (1998) hace una discusión interesante del tema desde el punto de vista de la diversificación. Primero, señala que "realmente no ha existido método alguno para medir el grado de diversificación en una cartera de deuda", y que "ex ante”, ningún método ha sido creado para cuantificar concentración; la concentración sólo ha sido detectada "ex post". Después, argumenta que "medir la diversificación de una cartera significa especificar el intervalo de posibles pérdidas y la probabilidad con la que se podrían dar". Más adelante, Kealhoffer provee una definición que permite la comparación del grado de diversificación de dos carteras como:

"La cartera A está mejor diversificada que la cartera B si ambas tienen la misma pérdida esperada, pero la probabilidad de que la pérdida exceda un cierto porcentaje es más pequeña para A que para B".

De esta manera, cuando se trata de préstamos bancarios tradicionales, ninguna metodología formal para medir la concentración parece emerger. Como dicen Altman y Saunders (1998), el tema de la medición de la concentración se ha tratado de manera subjetiva. Típicamente, los bancos y otros agentes aplican una técnica de calificación basada en la opinión de un grupo de expertos, sobre el grado de concentración observado en diferentes segmentos de la cartera, considerados con base en un criterio de clasificación, para obtener un indicador de la concentración de crédito. Generalmente, el número obtenido vale más en términos cardinales y jerárquicos, que como una medida directa del riesgo que pueda ser traducida rápidamente en pérdidas potenciales o en valor del riesgo ${ }^{10}$. No obstante, los resultados del ejercicio proveen los elementos principales para establecer límites sobre

\footnotetext{
${ }^{8}$ Véase Caouette, Altman, y Narayanan 1998, capítulos 17 y 18. Véase también Kealhofer 1998.

${ }^{9}$ Véase por ejemplo Bennet 1984.

${ }^{10}$ Véase por ejemplo Moody's Investor Services 1991, y el Informe de 1993 de Coopers y Lybrand.
} 
los créditos como una proporción del capital que puede destinarse a las diferentes áreas donde puede ocurrir la concentración.

La obtención de una buena medida ex-ante de concentración es difícil por varias razones. Primero, uno debe identificar o decidir las clases o tipos de concentración que son relevantes en una situación en particular (v.gr. por región geográfica, industria, mercados, productos, etc.). Aun si todos los créditos en estas dimensiones de posible concentración son relativamente pequeños en lo individual, pueden mostrar excesiva concentración en cualquier dimensión, si las probabilidades de impago están altamente correlacionadas o se correlacionan significativamente en circunstancias económicas adversas. Segundo, es necesario decidir la jerarquía adecuada dentro de cada clasificación en particular. Por ejemplo, qué es más importante: ¿Que el crédito sea una pequeña hipoteca o que el deudor viva en un pueblo en particular, o ambos?

Otro obstáculo es la cultura tradicional de los analistas de crédito, que casi por definición están altamente especializados. Esto hace difícil para estos analistas percibir el riesgo de la concentración, porque cada uno se dedica a una dimensión particular de la concentración. Además, el análisis que se aplica a créditos al consumo difiere significativamente del que se aplica para analizar un crédito para la construcción de una planta siderúrgica. No se emplean las mismas herramientas analíticas, ni los mismos criterios y ni siquiera el mismo argot. En cualquier caso, el lenguaje empleado generalmente no está asociado con los conceptos ni con los términos actualmente aceptados en la administración de riesgos. Mientras que los analistas de crédito al consumo infieren riesgos de cosas como la estabilidad en el trabajo y el ingreso familiar, los que analizan créditos industriales lo hacen en términos de razones financieras, flujos de efectivo descontados y garantías. Los administradores de riesgo en cambio discuten en términos de volatilidades, correlaciones, Griegas, perfiles de riesgo y valor en riesgo. De esta manera, aunque no hay duda de que hay analistas de crédito y administradores de riesgo que entienden los términos del otro, existe una brecha conceptual y de comunicación que necesita llenarse, para establecer un conjunto común de medidas de riesgo, que proporcione la asesoría integral necesaria para atender el tema de la concentración.

El enfoque adoptado en el análisis que sigue no resuelve todos estos problemas, pero sí provee un marco teórico que puede permitir la medición de la concentración ex-ante. La medida obtenida está relacionada directamente con el riesgo de crédito expresado en términos afines a los usados por los administradores de riesgos. Se verá que la medida de concentración obtenida es compatible con la noción de Kealhoffer antes citada. Partiendo de un modelo simple, donde se supone una sola dimensión de concentración y créditos con la misma probabilidad de incumplimiento, se desarrolla 
un modelo general donde, por lo menos teóricamente, se puede manejar cualquier número de dimensiones de concentración, y los créditos de cada segmento pueden tener probabilidades de incumplimiento diferentes, además de estar correlacionados entre sí.

\section{Valor en Riesgo, Concentración y el "Límite Individual": El Caso Simple.}

Tradicionalmente, los bancos tratan de atender el riesgo de concentración poniendo un límite a la cantidad máxima que puede prestarse a un solo deudor, a lo largo de las diferentes dimensiones donde se puede dar la concentración; esto es, por industria, región geográfica, productos, países, etc. En lo que sigue, se supone que la concentración ocurre en una sola dimensión, y la probabilidad de impago "p" de cualquier crédito es la misma para todos los créditos y cada uno es independiente de los demás. Normalmente, el "límite individual" se expresa como una proporción " $\delta$ " del capital "K" del banco. Sin embargo, al discutir la concentración del crédito, uno normalmente direcciona el tema a cuánto del crédito total está concentrado en un solo individuo o grupo pequeño de deudores. Así, cualesquiera que sean las virtudes de establecer límites como porcentaje del capital, ello no da mucha información sobre la concentración del crédito de una cartera. Para ver esto, nótese que por lo menos teóricamente, un banco puede tener todo el crédito otorgado a un solo deudor sin que este crédito exceda la proporción del capital que exige el límite pero bajo cualquier criterio representaría una cartera totalmente concentrada. Por otro lado, el banco puede tener un millón de créditos del mismo tamaño en cuyo caso su cartera está totalmente diversificada, independientemente de que cada crédito respete o no el límite. De esta manera, uno puede tener carteras muy concentradas así como carteras altamente diversificadas que respeten las restricciones en términos del capital ${ }^{11}$. Por estas razones se hace a un lado la tradición, ya que es mejor pensar en la concentración en términos de proporciones del valor total de la cartera de crédito, y cambiar el concepto de los límites. Así, para propósitos de concentración crediticia, se fijará el límite como una proporción " $\theta$ " del valor total de la cartera "V". Además, se puede comprobar fácilmente que $\delta$ y $\theta$ están relacionados linealmente a través de la razón de capitalización del banco, por lo que no se pierde generalidad. Para ver esto, sea $f_{k}$ el monto del "késimo" de "N" créditos de la cartera y nótese que:

$$
f_{k} \leq \delta K=\delta \frac{K}{V} \bullet V=\delta \psi V=\theta V ; k=0,1,2,3, \ldots ., \mathrm{N}
$$

\footnotetext{
${ }^{11}$ Por ejemplo, si en un banco con 100 millones de pesos de capital, los créditos están limitados a no exceder el $12 \%$ del capital, esto puede hacerse con solo un crédito de 12 millones, en cuyo caso la concentración es la máxima. Por otro lado, si la cartera tiene 100 mil créditos y cada uno es de 12 millones, ninguno excede el $12 \%$ del capital, pero se trata de una cartera altamente diversificada.
} 
donde $\psi=\frac{K}{V}$ es la razón de capitalización del banco. Así, $\theta=\delta \psi$, y el límite sobre el tamaño de los créditos se puede representar mediante la restricción siguiente:

$$
f_{k} \leq \theta V ; \quad k=0,1,2,3, \ldots, \mathrm{N}
$$

Si la concentración tiene que ver con el número de deudores que tienen más créditos, entonces la máxima concentración que puede tenerse respetando el límite, es cuando se concentra todo el crédito en un número mínimo de créditos " $n$ ”, que respeta la restricción previa, es decir:

$$
f_{k}=\left\{\begin{array}{l}
\theta V ; \mathrm{k}=0,1,2,3, \ldots ., \mathrm{n} \\
0 ; \mathrm{k}=\mathrm{n}+1, \mathrm{n}+2, \ldots, \mathrm{N}
\end{array}\right.
$$

En el siguiente argumento se supone que " $n$ " es siempre un entero y satisface ${ }^{12}$ :

$$
n \theta V=V \Rightarrow n \theta=1 \text { i.e. } n=1 / \theta
$$

Con base en estos supuestos, la probabilidad de que " $m$ " de los " $n$ " créditos dejen de pagarse se distribuye de acuerdo a una binomial; es decir:

$$
\operatorname{Pr}\{m ; n\}=\left(\begin{array}{l}
n \\
m
\end{array}\right) p^{m}(1-p)^{n-m}
$$

Es bien sabido que para una "n" grande, la distribución binomial se puede aproximar con una distribución normal, donde:

$$
\text { Media }=\mu=\mathrm{np} \text { y la Desviación Estándar } \sigma=\sqrt{\mathrm{np}(1-\mathrm{p})}
$$

Ahora, procediendo en forma actuarial, sea “ $\alpha$ ” el nivel de confianza adoptado y sea:

$$
n_{\alpha}=n p+z_{\alpha} \sqrt{n p(1-p)}
$$

Es decir: La probabilidad de que más de " $n \alpha$ " créditos dejen de pagar es " $\alpha$ ". En la expresión anterior, " $\mathrm{z}_{\alpha}$ " es la variable normal estandarizada que corresponde al nivel de confianza " $\alpha$ "

\footnotetext{
${ }^{12}$ No se pierde mucha generalidad bajo este supuesto, dado que el número de créditos es usualmente grande y $\theta$ está siempre bajo el control del administrador de riesgo. Relajar el supuesto no cambia el resultado y complica el álgebra innecesariamente.
} 
escogido. Ahora, si cada crédito vale " $\theta V$ ", lo anterior significa que el Monto en Riesgo para una cartera que exhibe este patrón de concentración, con nivel de confianza " $\alpha$ " es:

$$
V a R_{\alpha}=n_{\alpha} \theta V
$$

Si se quiere que la pérdida con nivel de confianza “ $\alpha$ ”, no exceda el capital " $K$ ”, entonces:

$$
\left\lfloor n p+z_{\alpha} \sqrt{n p(1-p)}\right\rfloor \theta V \leq K
$$

Despejando $\theta$ de lo anterior, y recordando que $\mathrm{n} \theta=1$, con un poco de manipulación algebraica se llega a:

$$
\theta \leq \frac{\left(\frac{K-p V}{z_{\alpha} V}\right)^{2}}{p(1-p)}=\frac{(\psi-p)^{2}}{z_{\alpha}^{2} p(1-p)}=\Theta(p, \psi, \alpha)
$$

En la expresión anterior, $\psi=\frac{K}{V}$ es la "razón de capitalización" del banco. Nótese que en estos términos, la suficiencia de capital existe si se satisface la desigualdad siguiente:

$$
\psi \geq \frac{\mathrm{VaR}_{\alpha}}{\mathrm{V}}=\mathrm{p}+\mathrm{z}_{\alpha} \sqrt{\mathrm{p}(1-\mathrm{p}) \theta}
$$

La expresión (3.5) es por demás atractiva, porque relaciona un indicador de concentración " $\theta$ ", con la razón de capitalización del banco " $\psi$ ", el riesgo de incumplimiento de los deudores individuales a través de la probabilidad de impago " $p$ ", y el monto en riesgo a través de " $\mathrm{z}_{\alpha}$ ". El límite individual de los créditos se asocia con el mínimo número de deudores donde se concentra todo el crédito $\mathrm{n}=\frac{1}{\theta}$, que es también un indicador de concentración, por lo que la cota obtenida también se puede tomar como un límite para la concentración de la cartera. La expresión (3.6) representa una relación de suficiencia de capital en términos del riesgo de crédito de la cartera, que explícitamente incluye una medida " $\theta$ " de la concentración de la cartera. Como se verá en este documento, estas expresiones son muy robustas bajo condiciones mucho más generales. 


\section{Una Primera Generalización.}

Evidentemente, el patrón de concentración descrito, en donde la cartera de crédito contiene exactamente " $n$ " créditos en el tope permitido, no es demasiado realista. Por tanto, es importante ver si se puede obtener un indicador de concentración, que tenga sentido en términos de monto en riesgo, y que al mismo tiempo permitiera un poco más de juego en la conformación de la cartera crediticia de un banco. Así, sea $\mathrm{F}=\left(f_{i}\right) \in \mathrm{E}^{N}$ el vector que representa la cartera crediticia de un banco $^{13}$, y sea $f_{i}=$ monto del i-ésimo crédito en la cartera; $i=1,2, \ldots, N$.

Si la probabilidad de impago de cada crédito es "p", y suponiendo independencia, podemos definir "N" variables aleatorias binarias "xi" tales que:

$$
x_{i}=\left\{\begin{array}{l}
f_{i} \text { con probabilidad } p \\
0 \text { con probabilidad 1-p }
\end{array}\right.
$$

Se sabe que $E\left(x_{i}\right)=p f_{i}$ y que la varianza de $x_{i}$ es $p(1-p) f_{i}^{2}$. Entonces, bajo el supuesto de independencia, se tiene que:
a) $\quad \mu=\mathrm{E}\left(\sum_{\mathrm{i}=1}^{\mathrm{N}} \mathrm{x}_{\mathrm{i}}\right)=\sum_{\mathrm{i}=1}^{\mathrm{N}} \mathrm{pf}_{\mathrm{i}}=\mathrm{pV}$; donde $V=\sum_{\mathrm{i}=1}^{\mathrm{N}} \mathrm{f}_{\mathrm{i}}$
b) $\sigma^{2}=\operatorname{VAR}\left(\sum_{\mathrm{i}=1}^{\mathrm{N}} \mathrm{x}_{\mathrm{i}}\right)=\sum_{\mathrm{i}=1}^{\mathrm{N}} \operatorname{VAR}\left(\mathrm{x}_{\mathrm{i}}\right)=\sum_{\mathrm{i}=1}^{\mathrm{N}} \mathrm{p}(1-\mathrm{p}) \mathrm{f}_{\mathrm{i}}^{2}=\mathrm{p}(1-\mathrm{p}) \sum_{\mathrm{i}=1}^{\mathrm{N}} \mathrm{f}_{\mathrm{i}}^{2}$

Al ser $F=\left(f_{i}\right)$ un vector arbitrario, es difícil conocer la distribución exacta de $\sum_{i=1}^{N} x_{i}$. Sin embargo, para una $\mathrm{N}$ grande, la distribución Normal debe ser una aproximación razonable ${ }^{14}$ y por tanto:

$$
\operatorname{VaR}_{\alpha}=\mu+z_{\alpha} \sigma=p V+z_{\alpha} \sqrt{p(1-p) \sum_{i=1}^{N} f_{i}^{2}}
$$

Siguiendo la tónica del análisis anterior, si se requiere que $V a R_{\alpha} \leq K$, después de un poco de manipulación algebraica se obtiene:

\footnotetext{
${ }^{13} \mathrm{E}^{\mathrm{N}}$ denota al espacio Euclidiano N-dimensional.

${ }^{14}$ Véase por ejemplo DeGroot (1988), pág. 263.
} 


$$
H(F)=\frac{\sum_{i=1}^{N} f_{i}^{2}}{\left(\sum_{i=1}^{N} f_{i}\right)^{2}} \leq \frac{(\psi-p)^{2}}{z_{\alpha}^{2} p(1-p)}=\Theta(p, \psi, \alpha)
$$

Nótese que la cota obtenida $\Theta(p, \psi, \alpha)$ es exactamente la misma que la que se obtuvo en el primer caso, cuando se supuso que la cartera crediticia consistía en " $n$ " créditos del mismo monto $\theta \mathrm{V}$. La gran diferencia, es que en lugar de usar el límite de crédito otorgable a un deudor como medida de concentración, ahora la concentración del crédito está medida por:

$$
\text { Concentración }=H(F)=\frac{\sum_{i=1}^{N} f_{i}^{2}}{\left(\sum_{i=1}^{N} f_{i}\right)^{2}}
$$

El lector familiarizado con la literatura técnica sobre concentración industrial habrá reconocido en el anterior al índice de Herfindahl-Hirschman ${ }^{15}$.

\section{Análisis de la Desigualdad de la Suficiencia de Capital.}

El primer punto importante a notar es que, con las limitaciones del caso, parece que el nivel de concentración de una cartera puede controlarse utilizando una medida general de concentración, aparte del límite individual del crédito. Después, es interesante notar que la suficiencia de capital representada por la razón de capitalización $\psi$ requiere que:

$$
\psi \geq p+z_{\alpha} \sqrt{p(1-p) H(\mathbf{F})}
$$

Esta desigualdad relaciona la suficiencia de capital con la probabilidad de impago, el nivel de confianza usado para calcular el valor en riesgo (VaR) y el índice de concentración. También muestra que existe una relación directa entre el índice de Herfindahl y la varianza de la probabilidad de impago p. Dado que el índice toma valores entre el recíproco del número de créditos "N" y uno,

\footnotetext{
${ }^{15}$ Véase por ejemplo Tirol 1995 y/o Shy 1995.
} 
cuando existe una alta concentración, la varianza $\sqrt{p(1-p) H(F)}$ variará entre $\sqrt{\frac{p(1-p)}{N}}$ y $\sqrt{p(1-p)}$, dependiendo del grado de concentración en la cartera medido por H(F). Además, nótese que el papel que juega $\mathrm{H}(\mathrm{F})$ es totalmente compatible con la definición de concentración de Kealhoffer dado que es obvio de (5.1) que para carteras con la misma pérdida esperada, entre menor sea el valor de $\mathrm{H}(\mathrm{F})$, menor será la probabilidad de que la pérdida exceda un nivel específico.

En lo que sigue, se verá que todo se comporta como debe. El teorema siguiente resume las principales implicaciones de las relaciones anteriores. El resultado se introduce temprano porque no cambia con las generalizaciones posteriores donde se relajan los supuestos de igualdad de probabilidades de impago e independencia, y porque muestra el poder del modelo para la administración de riesgos, la regulación y la supervisión bancaria.

\section{Teorema 5.1.}

La cota $\Theta(p, \psi, \alpha)$ sobre la medida de concentración tiene las siguientes propiedades:

1. $\Theta(p, \psi, \alpha)$ tiene una relación directa con la razón de capitalización $\psi$ y una relación inversa con la probabilidad de impago "p" y el nivel de confianza del valor en riesgo " $\mathrm{z}_{\alpha}$ ".

2. Si la medida de concentración excede la cota (i.e. $H(F)>\Theta(p, \psi, \alpha)$ ), entonces el capital del banco está en riesgo para el nivel de confianza escogido.

3. Si la probabilidad de impago "p" excede la razón de capitalización " $\psi$ ", entonces el capital del banco es insuficiente para enfrentar el riesgo asumido, para cualquier nivel de confianza y valor de la medida de concentración $\mathrm{H}(\mathrm{F})$.

4. Si $\Theta(p, \psi, \alpha)>1$, cualquier nivel de concentración es aceptable.

\section{Prueba.}

El punto uno es obvio por la forma de $\Theta(p, \psi, \alpha)$. El segundo punto se verifica fácilmente; esto es: Si H(F) $>\Theta(p, \psi, \alpha)$ entonces,

$$
\mathrm{VaR}_{\alpha}=\left(p+z_{\alpha} \sqrt{H(F) p(1-p)}\right) V>\left(p+z_{\alpha} \sqrt{\Theta p(1-p)}\right) V=\left(p+\frac{z_{\alpha} \sqrt{p(1-p)}(\psi-p)}{z_{\alpha} \sqrt{p(1-p)}}\right) V=K
$$


El punto tres sale directo de (5.1):

$$
\mathrm{VaR} \alpha \leq \mathrm{K} \Leftrightarrow \psi \geq \mathrm{p}+\mathrm{z}_{\alpha} \sqrt{\mathrm{p}(1-\mathrm{p}) \mathrm{H}(\mathrm{F})}
$$

Si $\mathrm{p}>\psi$, entonces se viola (5.1):

$$
\mathrm{VaR}_{\alpha}=\left(\mathrm{p}+\mathrm{z}_{\alpha} \sqrt{\mathrm{H}(\mathrm{F}) \mathrm{p}(1-\mathrm{p})}\right) \mathrm{V}>\left(\psi+\mathrm{z}_{\alpha} \sqrt{\mathrm{H}(\mathrm{F}) \mathrm{p}(1-\mathrm{p})}\right) \mathrm{V}=\mathrm{K}+\mathrm{z}_{\alpha} \mathrm{V} \sqrt{\mathrm{H}(\mathrm{F}) \mathrm{p}(1-\mathrm{p})}>\mathrm{K}
$$

Y para el punto cuatro, es bien sabido que $\mathrm{H}(\mathrm{F}) \leq 1$ para cualquier $\mathrm{F}^{16}$.

El Teorema 5.1 proporciona reglas útiles tanto para el administrador como para el regulador. Primero, permite determinar la suficiencia de capital además de que se pueden calcular con precisión los ajustes requeridos en la razón de capitalización por enfrentar variaciones en las tasas de incumplimiento y/o la concentración de la cartera de créditos. Además, dependiendo del control que los bancos tengan sobre la razón de impago y la concentración de los créditos, pueden calcularse también los ajustes necesarios en la probabilidad de impago y la concentración de la cartera de créditos. Así, si la concentración de la cartera de créditos excede la cota al nivel de confianza elegido, la desigualdad (4.2) proporciona una forma conveniente de hacer los ajustes necesarios en $\psi, p$ y $H(F)$ de manera que el riesgo de crédito no ponga en peligro el capital del banco. Otro resultado interesante, es que si la tasa de impago de la cartera excede la razón de capitalización, se está mandando una señal de alerta al administrador de riesgo y a las autoridades financieras, de que el capital del banco está en riesgo independientemente de la concentración de la cartera de crédito y el nivel de confianza elegido.

\section{El índice de Herfindahl: Dos Propiedades Importantes para Manejar el Riesgo de Concentración de Crédito.}

Los resultados anteriores proporcionan un marco analítico atractivo para determinar la suficiencia de capital, y medir la concentración en su relación con el riesgo de crédito. Una de las principales virtudes, es que surge de manera natural el índice de Herfindahl como medida de concentración, directamente relacionado con el riesgo de crédito. En particular, se muestra que bajo ciertos supuestos, el límite individual de crédito y el índice de Herfindahl son medidas de la concentración de la cartera que tienen la misma cota para propósitos de suficiencia de capital. Sin embargo quedan cabos sueltos, con respecto a cómo el índice de Herfindahl se relaciona con la idea intuitiva de que

\footnotetext{
${ }^{16}$ Ver Encaoua y Jacquemin 1980.
} 
la concentración está relacionada con el número mínimo de créditos donde existe mayor concentración. Además, dado que la concentración crediticia se administra y regula fijando límites a los créditos individuales, otra característica deseable de un índice de concentración, es que el valor del índice esté relacionado con el límite que se fije para el tamaño de los créditos.

Es conveniente adoptar la notación matricial-vectorial para simplificar el análisis. Entonces, se cambiará la notación como sigue:

$$
H(F)=\frac{\sum_{i=1}^{N} f_{i}^{2}}{\left(\sum_{i=1}^{N} f_{i}\right)^{2}}=\frac{\|F\|^{2}}{\left(1^{\top} F\right)^{2}}
$$

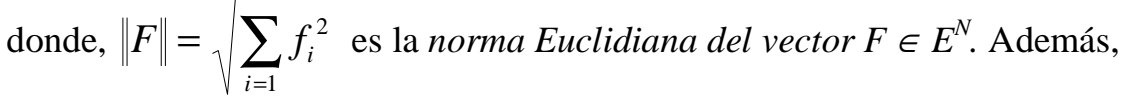

$$
I^{\top} \mathrm{F}=\sum_{\mathrm{i}=1}^{N} \mathrm{f}_{\mathrm{i}} \text { donde “ } 1 \text { ” denota el vector unitario en } E^{N} ; \text { esto es: } 1=\left(\begin{array}{c}
1 \\
1 \\
\cdot \\
\cdot \\
1
\end{array}\right)
$$

Nótese que $\mathbf{1}^{\mathrm{T}} F=V$ siempre, y puede considerarse un factor constante de normalización, de manera que lo que realmente diferencia la concentración en una cartera de crédito es $\|F\|$. Para examinar cómo la concentración se relaciona con la idea de que mucho crédito en pocas manos significa más concentración, se debe ser consistente con la idea de que la máxima concentración ocurre cuando todo el crédito está en un solo deudor y la mínima cuando todos los deudores deben el mismo monto. De manera formal:

En una cartera de "N" créditos,

a) La máxima concentración ocurre cuando para algún “i”, se tiene que:

$$
f_{j}= \begin{cases}V & \text { para } j=1 \\ 0 & \text { para } j \neq i ; j=1,2, \ldots N\end{cases}
$$

i.e. $\mathbf{F}^{\mathbf{m a x}}=\mathbf{V} \mathbf{e}^{\mathbf{i}}$, donde $\mathbf{e}^{\mathbf{i}} \in \mathbf{E}^{\mathbf{N}}$ es el i-ésimo vector unitario. 
b) La concentración mínima ocurre cuando $\mathrm{f}_{\mathrm{i}}=\frac{\mathrm{V}}{\mathrm{N}}$ para $i=1,2, \ldots, N$

El índice de Herfindahl-Hirschman ha sido estudiado ampliamente, principalmente en relación con la concentración industrial, y se sabe que tiene varias propiedades importantes. Así, es bien conocido que el índice toma valores entre el recíproco de " $\mathrm{N}$ " y uno ${ }^{17}$, y también se ha establecido que este índice se comporta bien en términos de "las cinco propiedades de las medidas de desigualdad ${ }^{18}$.

El índice de Herfindahl tiene varias propiedades interesantes relacionadas con números. La más conocida es la del "equivalente-numérico"19 de Adelman, que establece que su inverso puede interpretarse como "el mínimo número de créditos del mismo tamaño que resultarían en un valor específico del índice". En la primera obtención de la cota $\Theta(p, \psi, \alpha)$, basada en ideas puramente intuitivas, se estableció que la concentración máxima que se puede presentar, respetando el límite de crédito establecido, se da cuando se les presta hasta el límite al mínimo número posible de deudores. Se muestra ahora que el índice de Herfindahl es compatible con esta idea intuitiva; esto es: En una cartera en la que los créditos están restringidos a no exceder cierto límite, el valor del índice se maximiza cuando todo el crédito está concentrado en el mínimo número de acreditados, y cada acreditado está endeudado hasta el límite. El teorema establece la relación entre el límite individual de crédito y la medida de concentración Herfindahl-Hirshman, y al hacerlo, se muestra que el equivalente numérico es de hecho la máxima concentración posible, cuando los préstamos se restringen a cierto límite ${ }^{20}$.

\section{Teorema 6.1.}

Si se requiere que $F \leq \theta V 1$, entonces $H(F) \leq \theta$ y la máxima concentración bajo la medida de Herfindahl-Hirschman se da si y sólo si F es alguna permutación de la distribución siguiente:

\footnotetext{
${ }^{17}$ Es posible una normalización simple, donde fácilmente se ve que $\phi(F)$ como a continuación se define, satisface $0 \leq \phi \leq 1$

$$
\phi(F)=\frac{N-\frac{1}{H(F)}}{N-1}
$$

${ }^{18}$ Véase Cowell 1995 y Encaoua y Jacquemin 1980.

${ }^{19}$ Véase Adelman 1969 y Kelly Jr. 1981. Para otras propiedades interesantes numéricas relacionadas, véase Weinstock 1984.

${ }^{20}$ Aunque el resultado concuerda con la intuición, no se encontraron pruebas formales de los autores en referencias como Sleuwaegen et. al. 1989, Weinstock o Encaoua y Jaquemin op. cit..
} 


$$
\begin{aligned}
& f_{k}=\left\{\begin{array}{cl}
\theta V ; & k=0,1,2,3, \ldots, n \\
0 ; & k=n+1, n+2, \ldots ., N
\end{array}\right. \\
& \text { donde } n \theta V=V \Rightarrow n \theta=1 o \quad n=1 / \theta
\end{aligned}
$$

\section{Prueba:}

Por ser algo laboriosa, aquí se proporcionará solamente un esbozo de la prueba. Los detalles de la demostración del teorema se pueden consultar en el apéndice. Primero se prueba que la concentración máxima posible respetando el límite, se logra cuando el mínimo número posible de acreditados se endeuda hasta el tope $\theta \mathrm{V}$, y los demás tienen cero crédito. Determinar el máximo de $\mathrm{H}(\mathrm{F})$ respetando la restricción $\mathrm{F} \leq \theta \mathrm{V} \mathbf{1}$, equivale a encontrar una solución al problema de optimización siguiente:

$$
\begin{gathered}
\max \mathbf{F}^{\mathrm{T}} \mathbf{F} \\
\text { s.a.: } \mathbf{1}^{\mathrm{T}} \mathbf{F}=\mathrm{V} \\
\mathbf{F} \leq \boldsymbol{\theta} \mathbf{V} \mathbf{1} \\
\mathbf{F} \geq 0
\end{gathered}
$$

Para probar necesidad, se demuestra que la solución propuesta satisface las condiciones de KarushKuhn-Tucker para este problema. Para probar suficiencia, se argumenta que dado que se está maximizando una función convexa sujeta a restricciones lineales, la solución óptima del problema se encuentra en un punto extremo del poliedro convexo definido por las restricciones. Se puede ver que todos los puntos extremos de este poliedro son vectores con " $k$ " elementos iguales a $\theta V$, y los restantes son ceros; $k \leq n$. De esta manera el máximo se obtiene cuando $\mathrm{k}=\mathrm{n}$.

De aquí, es fácil verificar que $F \leq \theta V \boldsymbol{I}$ implica $H(F) \leq \theta$ como sigue: dado que la máxima concentración tiene exactamente "n" créditos en el límite $\theta \mathrm{V}$, entonces:

$$
\| F^{*}=\sqrt{\sum_{k=1}^{N}\left(f_{k}^{*}\right)^{2}}=\sqrt{\sum_{k=1}^{n}(\theta V)^{2}}=\sqrt{n} \theta V=\sqrt{\theta} V
$$

Como $n \theta=1$, esto significa que $H(F) \leq H\left(F^{*}\right)=\frac{\left\|F^{*}\right\|^{2}}{\left(1^{T} F^{*}\right)^{2}}=\frac{\theta V^{2}}{V^{2}}=\theta$ 
Este resultado tiene implicaciones importantes para la administración de riesgos y la regulación, de hecho, establece que poniendo un límite sobre los créditos individuales, se está aplicando también un límite a la concentración medida con el índice de Herfindahl en la misma cantidad $\theta$. De esta manera, es simple comprobar la suficiencia de capital comparando el parámetro individual $\theta$ con $\frac{(\psi-p)^{2}}{z_{\alpha}^{2} p(1-p)}=\Theta(p, \psi, \alpha)$. De forma alternativa, de (5.1), puede obtenerse la relación de suficiencia de capital en términos del límite individual del crédito (3.6); es decir:

$$
\psi \geq p+z_{\alpha} \sqrt{p(1-p) \theta}
$$

Así, (6.1) proporciona una regla de dedo simple para verificar la suficiencia de capital, sin hacer cálculos complicados. Por ejemplo, se toma $\theta$ como el cociente entre el crédito más grande que tiene la cartera y el valor total de ésta, y la tasa observada de impago como la aproximación ex post de la probabilidad de impago y se sustituyen estos valores en el lado derecho de (6.1). Como el Teorema (6.1) garantiza que $H(F) \leq \theta$, si la desigualdad se conserva es una buena señal de que el banco está bien capitalizado.

Sin embargo, debe tomarse en cuenta que esta condición es suficiente mas no necesaria. Como se mostrará en el siguiente teorema, si uno adopta la política de que la cartera debe satisfacer la restricción $H(\mathbf{F}) \leq \theta$, se pudiera dar un crédito que a la luz de la regla de límites se viera como “muy” grande. La prueba también es laboriosa ya que implica ver las condiciones KKT para un problema de optimización no convexo y también se relegó al apéndice para no distraer al lector del tema central de este documento. Aquellos interesados en los detalles pueden remitirse al apéndice a su conveniencia.

\section{Teorema 6.2.}

Si $\mathrm{H}(\mathbf{F}) \leq \theta$ entonces:

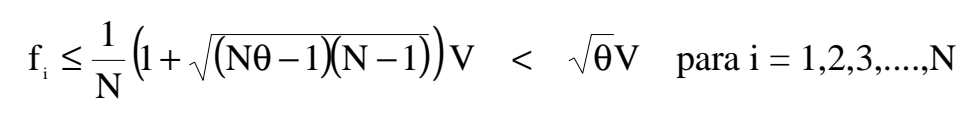

\section{Corolario:}

Sea $\alpha * \mathrm{~V}$ el crédito individual más grande que se puede tener bajo la restricción $\|a\|^{2} \leq \theta$. Entonces: 


$$
\begin{array}{ll}
\text { i) } \alpha^{*}= & \frac{1}{N}(1+\sqrt{(N \theta-1)(N-1)}) \\
\text { ii) } & \theta<\alpha^{*}<\sqrt{\theta} \\
\text { iii) } & H(F) \leq \theta \Rightarrow f_{k} *<\sqrt{\theta} V \forall \mathrm{k} .
\end{array}
$$

El corolario se afirma sin prueba, ya que es obvio el resultado por la demostración del teorema según se muestra en el apéndice. Lo interesante de este resultado está contenido en los incisos (ii) y (iii) del corolario, ya que bajo este criterio, la imposición de una restricción al índice de concentración acota el máximo crédito superior e inferiormente, y se sabe que ningún crédito puede exceder la cota superior, que en este caso es $\sqrt{\theta} V>\theta V$.

Es necesario enfatizar que las medidas de concentración que pueden ser apropiadas en algunos casos, no son necesariamente buenas en otros. Sin embargo, es deseable tener un buen índice de concentración, particularmente desde el punto de vista regulatorio, ya que facilita las comparaciones en términos de concentración de créditos entre diferentes instituciones, y conduce a una evaluación del riesgo de concentración del sistema financiero en su conjunto. Para el administrador de riesgos de un banco en particular, aparte de medir su propio riesgo, le provee de puntos de referencia para establecer estrategias de negocios y metas, y le permite hacer comparaciones con la competencia. Como se ha visto, el índice de Herfindahl parece ser muy conveniente dado que aparte de medir la concentración, está directamente relacionado con el riesgo y provee un medio rápido para comprobar la suficiencia de capital. En la siguiente sección se verá que el concepto es robusto bajo condiciones mucho más generales.

\subsection{Un ejemplo numérico.}

Para poder ver el significado de los resultados anteriores, a continuación se presenta un ejemplo

\begin{tabular}{|c|c|c|c|c|c|c|c|c|}
\hline \multirow{2}{*}{$\begin{array}{c}\mathbf{N}^{\circ} \text { de } \\
\text { Créditos }\end{array}$} & \multicolumn{7}{|c|}{ CALIFICACIÓN } & \multirow[b]{2}{*}{ TOTAL } \\
\hline & $\mathbf{A}$ & B & C & D & $E$ & $\mathbf{F}$ & $\mathbf{G}$ & \\
\hline 1 & $\$ 4,728$ & $\$ 5,528$ & $\$ 3,138$ & $\$ 5,320$ & $\$ 1,800$ & $\$ 1,933$ & $\$ 358$ & $\$ 22,805$ \\
\hline 2 & $\$ 7,728$ & $\$ 5,848$ & $\$ 3,204$ & $\$ 5,765$ & $\$ 5,042$ & $\$ 2,317$ & $\$ 1,090$ & $\$ 30,994$ \\
\hline 3 & & & $\$ 4,831$ & $\$ 20,239$ & $\$ 15,411$ & $\$ 2,411$ & $\$ 2,652$ & $\$ 45,544$ \\
\hline 4 & & & $\$ 4,912$ & & & $\$ 2,598$ & $\$ 4,929$ & $\$ 12,439$ \\
\hline 5 & & & $\$ 5,435$ & & & & $\$ 6,467$ & $\$ 11,902$ \\
\hline 6 & & & & & & & $\$ 6,480$ & $\$ 6,480$ \\
\hline TOTAL & $\$ 12,456$ & $\$ 11,376$ & $\$ 21,520$ & $\$ 31,324$ & $\$ 22,253$ & $\$ 9,259$ & $\$ 21,976$ & $\$ 130,164$ \\
\hline
\end{tabular}
numérico. Considérese la siguiente cartera de 25 créditos tomada del manual de CreditRisk $^{+}$:

Cuadro 6.1 
Las probabilidades de impago para los créditos se toman de la siguiente tabla:

\section{Cuadro 6.2.}

\begin{tabular}{|l|ccccccc|}
\hline Calificación & A & B & C & D & E & F & G \\
\hline $\begin{array}{l}\text { Probabilidad } \\
\text { de Impago.(\%) }\end{array}$ & 1.65 & 3.00 & 5.00 & 7.50 & 10.00 & 15.00 & 30.00 \\
\hline
\end{tabular}

Hasta este momento, el modelo sólo permite considerar carteras de crédito en donde la probabilidad de incumplimiento es la misma para todos los créditos. Así, para los propósitos de este ejercicio, supóngase que se toma como probabilidad de incumplimiento, el promedio ponderado de las probabilidades de incumplimiento mostradas en la tabla 6.2; que es $10.89 \%$.

El índice $\mathrm{HH}$ de la cartera es $6.61 \%$. Suponiendo normalidad y escogiendo un nivel de confianza de $5 \%, z \alpha=1.96$ se obtiene:

$$
\psi \geq p+z_{\alpha} \sqrt{p(1-p) H(F)}=0.1089+1.96 \sqrt{0.1089 \times .8911 \times 0.0661}=0.2658
$$

Esto significa que el capital económico del banco debe ser cuando menos de:

$$
\operatorname{VaR}_{.05}=0.2658 \times V=0.2658 \times \$ 130,164.00=\$ 34,602.79
$$

Supóngase que el capital económico es $\$ 35,000$, entonces la razón de capitalización es:

$$
\psi=\frac{K}{V}=\frac{35,000}{130,164}=0.2689
$$

Como $0.2689>0.2658$, el banco tiene suficiencia de capital. Ahora bajo (4.2), la máxima concentración admisible para la cartera sería:

$$
\frac{(\psi-p)^{2}}{z_{\alpha}^{2} p(1-p)}=\frac{(0.2689-0.1089)^{2}}{1.96^{2} \times 0.1089 \times 0.8911}=0.0687
$$

Dado que $\mathrm{H}(\mathrm{F})=6.61 \%$, la cartera no presenta una concentración excesiva. Finalmente, si el valor máximo del índice es $6.87 \%$, según el Teorema 6.2, ningún crédito de la cartera puede ser mayor que:

$$
f^{*}=\sqrt{0.0687} \times V=0.2621 \times 130,164=34,107.88
$$


El Cuadro 6.1 muestra que el crédito más grande en la cartera es el de calificación " $D$ " y monto igual a $\$ 20,239$, que es efectivamente inferior a la cantidad anterior.

Es interesante ver cual sería el tamaño máximo de crédito admisible, si se quisieran limitar los créditos a la cota de concentración. De acuerdo a 3.5, los créditos no pueden exceder:

$$
f_{i} \leq 0.0687 \times 130,164=\$ 8,942.27
$$

De hecho hay dos créditos considerablemente mayores que esta cantidad; el de $\$ 20,239$ y el de monto igual a \$15,411 con calificación "E". Esto pone en evidencia que la condición solamente es suficiente más no necesaria para evitar una concentración excesiva. Finalmente, se verifica que las cotas proporcionadas por el Teorema 6.2, se cumplen para el crédito más grande: $\$ 8,942.27 \leq$ $\$ 20,239 \leq \$ 34,107.88$.

\section{La Inclusión Explícita de Correlación de Incumplimientos}

Los resultados obtenidos hasta ahora dependen de los siguientes supuestos:

a) Las probabilidades de impago son homogéneas e independientes entre sí, para todos los créditos en la dimensión donde la concentración del crédito puede ocurrir.

b) Sólo hay una dimensión de la posible concentración del crédito.

c) Nada se recupera de los créditos que caen en impago.

Excepto en casos muy particulares, como para ciertas arrendadoras o sociedades de ahorro y préstamo, ninguno de los supuestos es totalmente realista. En esta sección, se relajan los supuestos y los resultados obtenidos se generalizan uno por uno. La primera generalización relaja completamente el supuesto de homogeneidad para examinar el caso donde las probabilidades de impago pueden ser diferentes y estar correlacionadas.

\subsection{Un Modelo General.}

Considérese el caso general donde todos los créditos tienen diferentes probabilidades de impago, y que además están correlacionados entre sí. Supóngase que la distribución de probabilidad de impago se puede caracterizar mediante un vector de "probabilidades esperadas de impago $\pi$ " y la correspondiente matriz de "varianza-covarianza M". La relación del monto en riesgo queda como sigue: 


$$
\mathrm{VaR}_{\alpha}=\pi^{\mathrm{T}} \mathbf{F}+\mathrm{z}_{\alpha} \sqrt{\mathbf{F}^{\mathrm{T}} \mathbf{M} \mathbf{F}} \leq \mathrm{K}
$$

Como M es positiva definida, existe una matriz "Q" tal que,

$$
\mathbf{M}=\mathbf{Q} \Lambda \mathbf{Q}^{T}
$$

donde $\Lambda$ es la matriz diagonal de valores característicos de $\mathbf{M}$ y $\mathbf{Q}$ es una matriz ortogonal de los vectores característicos de $\mathrm{M}$, con la propiedad de que $\mathbf{Q}^{-1}=\mathbf{Q}^{\mathbf{T} .21}$. Sea $\mathbf{S}=\mathbf{Q} \sqrt{\Lambda} \mathbf{Q}^{T}$, donde $\sqrt{\Lambda}$ es la matriz diagonal formada con las raíces cuadradas de los valores característicos de $\mathrm{M}$, de manera que $\mathbf{M}=\mathbf{S}^{\mathbf{T}} \mathbf{S}$. Ahora se hace el cambio de variable $\mathbf{G}=\mathbf{S F}$ para obtener:

$$
\mathrm{VaR}_{\alpha}=\pi^{\mathrm{T}} \mathbf{F}+\mathrm{z}_{\alpha} \sqrt{\mathbf{G}^{\mathrm{T}} \mathbf{G}} \leq \mathrm{K}
$$

Al multiplicar y dividir $\mathbf{G}^{\mathrm{T}} \mathbf{G}$ por $\left(\mathbf{1}^{\mathrm{T}} \mathbf{G}\right)^{\mathbf{2}}$, y dividir todo por el valor de la cartera $\mathrm{V}$, se obtiene:

$$
\bar{p}+z_{\alpha} \sigma \sqrt{H(\mathbf{G})} \leq \psi \quad \mathbf{y} \quad H(\mathbf{G}) \leq\left(\frac{\psi-\bar{p}}{z_{\alpha} \sigma}\right)^{2} \ldots(\mathbf{7 . 3 )}
$$

En lo anterior,

$$
\bar{p}=\frac{\pi^{T} \mathbf{F}}{V}
$$

es la probabilidad esperada de impago de la cartera y

$$
\sigma=\frac{\mathbf{1}^{T} \mathbf{G}}{V}=\frac{\mathbf{1}^{\mathrm{T}} \mathbf{G}}{\mathbf{1}^{\mathrm{T}} \mathbf{F}}
$$

es una medida de la desviación estándar. De hecho, las desigualdades de (7.3) tienen exactamente la misma forma que (4.2) y (5.1) excepto que ahora, el índice de Herfindahl se calcula sobre "G" en lugar de hacerse directamente sobre "F". Este cambio de variable efectuado equivale a un redimensionamiento de "F" en función de la matriz "S" que representa la "raíz cuadrada" de la matriz de varianza-covarianza "M". En términos de riesgo, lo que esto significa es que los créditos

\footnotetext{
${ }^{21}$ Se puede consultar cualquier libro de texto de álgebra lineal de nivel intermedio. Véase por ejemplo Strang G. 1980 .
} 
de la cartera se redimensionan de acuerdo a las covarianzas entre las probabilidades de impago de cada crédito. Es fácil verificar que lo anterior se reduce al caso simple unidimensional en donde las probabilidades de incumplimiento son iguales e independientes ${ }^{22}$ para todos los deudores.

Debe notarse que este redimensionamiento implica que la concentración en números no es lo único que debe tomarse en cuenta. Si bien mucho crédito en pocas manos puede ser riesgoso, seguramente es aún más peligroso que mucho riesgo esté concentrado en un grupo de créditos. Visto de otra manera, en un momento dado puede representar más riesgo un grupo de muchos créditos pequeños pero de varianzas grandes y altamente correlacionadas entre sí, que un pequeño grupo de créditos grandes, cuya varianza y probabilidades de impago sean pequeñas y que además no estén muy correlacionadas ni entre sí, ni con otros créditos. El redimensionamiento de "F" a través de "S", proporciona los medios para abordar este problema. Al redimensionar el vector de créditos, la varianza total de las pérdidas $\sigma \sqrt{H(\mathbf{G})}$, se descompone en el efecto de la varianza de las probabilidades de incumplimiento, medida por $\boldsymbol{\sigma}$ y el efecto de la concentración medida por $H(G)$.

\subsection{La Determinación de Límites Individuales sobre los Créditos y Suficiencia de Capital.}

Como se ha mencionado, la forma típica de administrar la excesiva concentración de crédito es fijando límites a la cantidad de crédito que se le puede otorgar a un deudor. Aunque el Teorema 6.1 indica que $\mathbf{G} \leq \theta \mathbf{1}\left(\mathbf{1}^{\mathrm{T}} \mathbf{G}\right)$ implica $\mathrm{H}(\mathbf{G}) \leq \theta$, es difícil de instrumentar. Para propósitos prácticos, los límites que son útiles son aquellos que están puestos directamente sobre $\mathbf{F} .^{23}$

Por ejemplo, dado que $\mathbf{F} \leq \theta \mathbf{1 V}$ implica $\mathrm{H}(\mathbf{F}) \leq \theta$ por el Teorema 6.1 entonces:

$$
\mathrm{H}(\mathbf{G}) \leq \theta \frac{\mathrm{H}(\mathbf{G})}{\mathrm{H}(\mathbf{F})} \leq\left(\frac{\psi-\overline{\mathrm{p}}}{\mathrm{z}_{\alpha} \sigma}\right)^{2}
$$

Entonces

$$
\theta \leq \frac{\mathrm{H}(\mathbf{F})}{\mathrm{H}(\mathbf{G})}\left(\frac{\psi-\overline{\mathrm{p}}}{\mathrm{z}_{\alpha} \sigma}\right)^{2}
$$

\footnotetext{
${ }^{22}$ Simplemente hágase $\boldsymbol{\pi}=\mathbf{p} \mathbf{1}$ y $\mathrm{M}=\mathbf{p}(\mathbf{1}-\mathbf{p}) \mathrm{I}$.

${ }^{23}$ Quisiéramos señalar que $\mathrm{F} \leq \theta \mathrm{VS}^{-1} \mathbf{1}$, presenta complicaciones técnicas serias ya que, entre otras cosas, la desigualdad no se cumple para cualquier $\mathrm{S}$.
} 
Nótese que $\left(\frac{\|\mathbf{G}\|}{\|\mathbf{F}\|}\right)^{2}$ es el "Cociente de Rayleigh", y es bien sabido que $\lambda_{\min } \leq\left(\frac{\|\mathbf{G}\|}{\|\mathbf{F}\|}\right)^{2} \leq \lambda_{\max }$, donde $\lambda_{\min }$ y $\lambda_{\max }$ son los valores característicos menor y mayor de la matriz M. Así, después de un poco de álgebra, por (7.5) se verifica que:

$$
\theta \leq \frac{1}{\lambda_{\max }}\left(\frac{\mathbf{1}^{\mathrm{T}} \mathbf{G}}{\mathbf{1}^{\mathrm{T}} \mathbf{F}}\right)^{2}\left(\frac{\psi-\bar{p}}{z_{\alpha} \sigma}\right)^{2}=\frac{1}{\lambda_{\max }}\left(\frac{\psi-\bar{p}}{z_{\alpha}}\right)^{2}
$$

también es una cota para el límite individual, aunque más estricta que (7.6) (Véase la nota de pie de la página siguiente). Por tanto, si se escoge $\theta$ que satisfaga (7.6) o (7.7) se garantiza que $\mathbf{F} \leq \theta \mathbf{1 V}$ implica el cumplimiento de (7.3).

Finalmente, se puede comprobar fácilmente que con la transformación de "F" a través de "S", no se afectan los resultados del Teorema 5.1, así como sus implicaciones regulatorias y de administración de riesgos. Específicamente, en lo que se refiere a la suficiencia de capital, la desigualdad (7.3), se generaliza a:

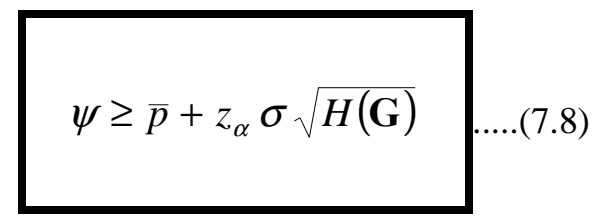

Además, (7.7) tiene una analogía con (6.1) donde la suficiencia de capital se relaciona con el límite individual de la forma siguiente:

$$
\psi \geq \bar{p}+z_{\alpha} \sqrt{\lambda_{\max } \theta}
$$

Es interesante notar que la única diferencia entre (7.9) y (6.1) es que aparece el valor característico mayor de "M" en la expresión. Se puede demostrar que $\lambda_{\max } \theta \geq \sigma 2 H(G)$, y que éste crece con correlación positiva, lo cual es congruente con la noción intuitiva de que el aumento en la correlación, incrementa el requisito de capitalización ${ }^{24}$. Nótese que (7.8) y (7.9) implican que la suficiencia de capital puede evaluarse con cuatro parámetros.

\footnotetext{
${ }^{24}$ Simplemente divídase (7.6) entre (7.7) y utilícense las cotas sobre el cociente de Rayleigh para llegar a la conclusión de que la relación es mayor que uno.
} 


\section{Ejemplo 7.1}

Para ilustrar los resultados anteriores, considérese el grupo de créditos del ejemplo anterior; Cuadro 6.1.

\section{Cuadro 7.1.}

\begin{tabular}{|l|l|ccccccc|}
\hline \multicolumn{2}{|c|}{ Calificación } & A & B & C & D & E & F & G \\
\hline $\begin{array}{l}\text { Probabilidad } \\
\text { de Impago (\%) }\end{array}$ & Media (p) & 1.65 & 3.00 & 5.00 & 7.50 & 10.00 & 15.00 & 30.00 \\
\hline
\end{tabular}

Por su tamaño, la matriz de covarianzas $\mathbf{M}$, se segmentó en tres bloques ${ }^{25}$.

$$
\mathbf{M}=\left[\begin{array}{lll}
\mathbf{M}_{1} & \mathbf{C}_{1,2} & \mathbf{C}_{1,3} \\
\mathbf{C}_{2,1} & \mathbf{M}_{2} & \mathbf{C}_{2,3} \\
\mathbf{C}_{3,1} & \mathbf{C}_{3,2} & \mathbf{M}_{3}
\end{array}\right]
$$

Factorizando la matriz para obtener $\mathbf{S}=\mathbf{Q} \sqrt{\Lambda} \mathbf{Q}^{T}$ y haciendo el cambio de variable $\mathbf{G}=\mathbf{S F}$, se obtiene el vector de créditos redimensionado, el cual se compara en la tabla 7.2 con el original. Nótese que, dado que el valor característico más grande de $\mathbf{M}$ es $\lambda_{\max }=0.8758<1$, “G” es una contracción de "F", y además, sus elementos están mejor distribuidos de manera que presenta menor concentración: $H(G)=4.57 \%<H(F)=6.61 \%$.

Así, aunque aun es cierto que el crédito "D" de \$20,239 y el crédito "E" de $\$ 15,411$, son los más grandes en ambos vectores, los dos disminuyen considerablemente de tamaño. Del Cuadro 7.2, nótese que hay elementos que disminuyen en tamaño y mientras que otros aumentan. En resumen, mientras que los créditos calificados de $A$ a $D$ representan una proporción menor en la cartera redimensionada en comparación con la original. Todos los créditos calificados como $F$ o menores, toman valores proporcionalmente mayores, y por tanto pesan más en la cartera redimensionada. Pareciera que los créditos calificados como $E$ representan una línea divisoria, donde los dos más grandes (12 y 13) se reducen en tamaño, pero el más pequeño (21), aumenta.

\footnotetext{
${ }^{25}$ Dichos bloques se presentan en las tablas B.2 a B.7 en el apéndice B.
} 
Cuadro 7.2

\begin{tabular}{|r|r|r|r|r|r|}
\hline & Calificación & \multicolumn{1}{|c|}{$\mathbf{F}$} & proporción & G & proporción \\
\hline 1 & $\mathrm{~A}$ & 4,728 & $3.6 \%$ & 1,660 & $1.7 \%$ \\
2 & $\mathrm{C}$ & 3,204 & $2.5 \%$ & 2,401 & $2.4 \%$ \\
3 & $\mathrm{C}$ & 4,912 & $3.8 \%$ & 2,738 & $2.8 \%$ \\
4 & $\mathrm{D}$ & 5,320 & $4.1 \%$ & 3,327 & $3.4 \%$ \\
\hline \hline $\mathbf{5}$ & $\mathrm{D}$ & $\mathbf{2 0 , 2 3 9}$ & $\mathbf{1 5 . 5} \%$ & $\mathbf{6 , 8 8 6}$ & $\mathbf{6 . 9} \%$ \\
\hline \hline 6 & $\mathrm{~F}$ & 1,933 & $1.5 \%$ & 3,216 & $3.2 \%$ \\
7 & $\mathrm{~F}$ & 2,598 & $2.0 \%$ & 3,431 & $3.5 \%$ \\
8 & $\mathrm{G}$ & 1,090 & $0.8 \%$ & 3,539 & $3.6 \%$ \\
9 & $\mathrm{~B}$ & 5,528 & $4.2 \%$ & 2,606 & $2.6 \%$ \\
10 & $\mathrm{C}$ & 3,138 & $2.4 \%$ & 2,761 & $2.8 \%$ \\
11 & $\mathrm{C}$ & 4,831 & $3.7 \%$ & 3,085 & $3.1 \%$ \\
12 & $\mathrm{E}$ & 5,042 & $3.9 \%$ & 4,081 & $4.1 \%$ \\
\hline \hline $\mathbf{1 3}$ & $\mathrm{E}$ & $\mathbf{1 5 , 4 1 1}$ & $\mathbf{1 1 . 8} \%$ & $\mathbf{6 , 8 1 0}$ & $\mathbf{6 . 9} \%$ \\
\hline \hline 14 & $\mathrm{~F}$ & 2,411 & $1.9 \%$ & 3,877 & $3.9 \%$ \\
15 & $\mathrm{G}$ & 358 & $0.3 \%$ & 3,845 & $3.9 \%$ \\
16 & $\mathrm{G}$ & 6,467 & $5.0 \%$ & 6,302 & $6.4 \%$ \\
17 & $\mathrm{~A}$ & 7,728 & $5.9 \%$ & 2,292 & $2.3 \%$ \\
18 & $\mathrm{~B}$ & 5,848 & $4.5 \%$ & 2,759 & $2.8 \%$ \\
19 & $\mathrm{C}$ & 5,435 & $4.2 \%$ & 3,367 & $3.4 \%$ \\
20 & $\mathrm{D}$ & 5,765 & $4.4 \%$ & 4,039 & $4.1 \%$ \\
21 & $\mathrm{E}$ & 1,800 & $1.4 \%$ & 3,619 & $3.7 \%$ \\
22 & $\mathrm{~F}$ & 2,317 & $1.8 \%$ & 4,303 & $4.3 \%$ \\
23 & $\mathrm{G}$ & 2,652 & $2.0 \%$ & 5,339 & $5.4 \%$ \\
24 & $\mathrm{G}$ & 4,929 & $3.8 \%$ & 6,143 & $6.2 \%$ \\
25 & $\mathrm{G}$ & 6,480 & $5.0 \%$ & 6,665 & $6.7 \%$ \\
\hline & & $\mathbf{1 3 0 , 1 6 4}$ & & $\mathbf{9 9 , 0 9 3}$ & \\
\hline
\end{tabular}

Suponiendo normalidad, con un nivel de confianza de 5\%, el valor en riesgo o monto en riesgo de la cartera es:

$$
\mathrm{VaR}_{.05}=\pi^{\mathrm{T}} \mathrm{F}+\mathrm{z} .05 \sqrt{\mathrm{F}^{\mathrm{T}} \mathrm{MF}}=14,179+1.96(21,176)=\$ 55,684
$$

Así, el capital mínimo requerido es de $\$ 55,684$ de manera que los $\$ 35,000$ supuestos como capital en el ejemplo 6.1, serían insuficientes para cubrir este nivel de riesgo.

Calculando ahora:

$$
\bar{p}=\frac{\boldsymbol{\pi}^{\mathrm{T}} \mathbf{F}}{V}=\frac{14,179}{130,164}=0.1089 \text { y } \sigma=\frac{\mathbf{1}^{\mathrm{T}} \mathbf{G}}{\mathbf{1}^{\mathrm{T}} \mathbf{F}}=\frac{99,093}{130,164}=0.7613
$$

La suficiencia de capital debe cumplir la desigualdad:

$$
\psi>p+z_{\alpha} \sigma \sqrt{H(G)}=0.4278
$$


Supóngase que $\mathrm{K}=\$ 60,000$, de manera que $\psi=60,000 / 130,164=0.461$. Entonces:

$$
\left(\frac{\psi-\bar{p}}{z_{\alpha} \sigma}\right)^{2}=\left(\frac{0.461-0.1089}{1.96(0.7613)}\right)^{2}=0.05568
$$

Al calcular el límite individual como proporción del valor de la cartera, de la ecuación (7.6), se obtiene:

$$
\theta \leq \frac{H(F)}{H(G)}\left(\frac{\psi-\bar{p}}{z_{\alpha} \sigma}\right)^{2}=\frac{0.0661}{0.0457}(0.05568)=0.0805
$$

Esto es:

$$
f_{i} \leq 0.0805 \times \$ 130,164=\$ 10,482.97
$$

Sólo existen dos créditos que exceden este límite (Cuadro 7.2).

Utilizando el cociente de Rayleigh, de (7.7) se obtiene:

$$
f_{i} \leq \theta V \leq \frac{1}{\lambda_{\max }}\left(\frac{\psi-\bar{p}}{z_{\alpha}}\right)^{2} V=\frac{1}{0.8758}\left(\frac{0.461-0.1089}{1.96}\right)^{2}(\$ 130,164)=\$ 4,796.29
$$

Como era de esperarse, este límite es mucho más restrictivo que el anterior; de hecho, es menos de la mitad del obtenido previamente. La mayoría de los créditos en la cartera sobrepasan este límite.

Debe notarse que la cartera de este ejemplo es muy mala, dado que existe una gran proporción de créditos con probabilidades de impago altas. Esto explica el requerimiento tan alto de capital de casi 43\% del valor de la cartera. Por ello resulta interesante comparar la desviación estándar de impago de los créditos correlacionados $\sigma \sqrt{H(G)}=0.1627$, con $\sqrt{p(1-p) H(F)}=0.0801$ de los créditos que suponen independencia en los impagos. Nótese que la correlación duplica la desviación estándar de la distribución de pérdidas de los créditos no correlacionados, de tal manera que el requerimiento de capitalización de $43 \%$ de la cartera con créditos correlacionados, es substancialmente mayor que el $26.6 \%$ requerido bajo el supuesto de independencia. 


\subsection{El Tratamiento de Diferentes Dimensiones de Concentración}

Generalmente, los bancos clasifican sus carteras de créditos en "segmentos" de acuerdo a algún criterio práctico de clasificación, como región geográfica, industria, o productos. Otro criterio es el de separar créditos al consumo por nivel de ingreso de los deudores, o créditos corporativos o empresariales por negocios de diferentes tamaños, y la clasificación responde de alguna manera a la forma en que los bancos hacen sus negocios. Para el propósito de riesgo de crédito en general y de concentración en particular, se desearía adoptar un criterio diferente. Como se mencionó, uno de los problemas más difíciles es determinar ex-ante, dimensiones potencialmente peligrosas de concentración, y eso puede no tener nada que ver con la estructura organizacional del banco. Afortunadamente, el modelo desarrollado permite una segmentación totalmente arbitraria de la cartera, de manera que puede analizarse desde varios ángulos, y permite determinar los segmentos donde la concentración es potencialmente riesgosa. Esto permite la distinción de límites para cada segmento, y la evaluación de las implicaciones en términos de la suficiencia de capital. En lo que sigue, se emplearán las palabras clase, segmento y dimensión de concentración como sinónimos.

\subsubsection{El Análisis de Segmentos Individuales.}

Supóngase que se hace una partición arbitraria de $F$ en $h$ clases, $F^{T}=\left(F_{1}, \ldots, F_{h}\right)$, donde $F_{i}$ es un vector que contiene los saldos de los créditos que pertenecen al $i$-ésimo segmento. Ahora se parten el vector de probabilidades esperadas de incumplimiento y la matriz de varianza-covarianza, de esta manera:

a) $\pi=\left(\pi_{i}\right)$; Partición del vector de probabilidades de impago, donde “ $\pi_{i}$ " es el vector de probabilidades de impago del segmento $\mathrm{i} ; \mathrm{i}=1,2,3, \ldots ., \mathrm{h}$

b) La matriz de varianza-covarianza se parte como:

$$
\mathbf{M}=\left[\begin{array}{cccc}
M_{1} & C_{12} & \cdots & C_{1 h} \\
C_{21} & M_{2} & \cdots & C_{2 n h} \\
\vdots & \vdots & \ddots & \vdots \\
C_{h 1} & C_{h 2} & \cdots & M_{h}
\end{array}\right]
$$

Cada submatríz $M_{i}$ corresponde a la matriz de varianza-covarianza idiosincrática del grupo "i” y tiene dimensión $\left(\mathrm{N}_{\mathrm{i}} \times \mathrm{N}_{\mathrm{i}}\right)$; donde $\mathrm{N}_{\mathrm{i}}$ es el número de créditos en el segmento. Todas estas matrices 
son positivas definidas al igual que $\mathbf{M}$ y se puede obtener su correspondiente factorización $\mathbf{S}_{\mathbf{i}}$. Las matrices " $\mathrm{C}_{\mathrm{ij}}$ " contienen las covarianzas de las probabilidades de impago entre los créditos del grupo “ $i$ ” y las del grupo “ $j$ ”.

A partir de aquí, sea $V_{i}=\sum_{j \in F_{i}} f_{j}$, el valor de la cartera asociada a la clase " $i$ ”, y $\sum_{i=1}^{h} V_{i}=V$. Ahora, sea $K_{i}=\gamma_{i} K$, donde " $\gamma_{i}$ " es la proporción del capital asignado al segmento " $i$ "; $\gamma_{i} \in[0,1] \forall i ; \sum_{i=1}^{h} \gamma_{i}=1$. Procediendo de la manera usual, la desigualdad en el valor en riesgo para cada clase es:

$$
V a R_{\alpha}^{i}=\boldsymbol{\pi}_{\mathbf{i}}^{\mathrm{T}} \mathbf{F}_{i}+z_{\alpha} \sqrt{\mathbf{F}^{T} \mathbf{R}_{i} \mathbf{F}} \leq K_{i}=\gamma_{i} K
$$

En lo anterior, es importante notar que la matriz " $R_{i}$ ” tiene la forma siguiente:

$$
\mathbf{R}_{\mathbf{i}}=\left[\begin{array}{ccccc}
0 & \cdots & C_{1 i} & \cdots & 0 \\
\vdots & \cdots & \vdots & \cdots & \vdots \\
C_{i 1} & \cdots & M_{i} & \cdots & C_{i N} \\
\vdots & \cdots & \vdots & \cdots & \vdots \\
0 & \cdots & C_{N i} & \cdots & 0
\end{array}\right] \ldots(\text { (7.11) }
$$

Cada matriz " $\mathbf{R}_{\mathrm{i}}$ " solamente toma en cuenta las correlaciones entre las probabilidades de impago de los créditos del grupo “i” con los de los demás grupos, pero elimina las correlaciones entre los demás grupos que no inciden directamente sobre el grupo en cuestión. Dividiendo ambos lados por el valor de la cartera $V_{i}$, y haciendo el cambio de variable $\mathbf{G}_{\mathrm{i}}=\mathbf{S}_{\mathrm{i}} \mathbf{F}_{\mathrm{i}}$, después de un poco de álgebra se llega a:

$$
H\left(\mathbf{G}_{\mathrm{i}}\right) \leq\left(\frac{\Psi_{\mathrm{i}}-\overline{\mathrm{p}}_{\mathrm{i}}}{\mathrm{z}_{\alpha} \sigma_{\mathrm{i}}}\right)^{2}-\frac{2}{\left(\mathbf{1}^{\mathrm{T}} \mathrm{G}_{\mathrm{i}}\right)^{2}} \sum_{\{\mathrm{j} j \neq i\}} \mathrm{F}_{\mathrm{i}}^{\mathrm{T}} \mathrm{C}_{\mathrm{ij}} \mathrm{F}_{\mathrm{j}} \ldots
$$

donde $\sigma_{i}=\frac{\mathbf{1}_{\mathbf{i}}^{\mathbf{T}} \mathbf{G}_{\mathbf{i}}}{V_{i}}$.

Resulta interesante notar que la cota de la concentración ahora incluye una corrección que tiene que ver con la correlación de impago con los créditos de otros segmentos (el segundo término en el lado 
derecho de la desigualdad). Esto concuerda con la intuición, dado que una correlación alta de impago con los créditos de otros segmentos, indica que se puede tolerar menos concentración en el grupo "i".

Con un argumento análogo al de la sección 7.2, se pueden obtener límites individuales $\theta_{i}$ para los créditos de cada segmento como:

$$
\theta_{i} \leq \frac{H\left(F_{i}\right)}{H\left(G_{i}\right)}\left\{\left(\frac{\psi_{i}-\bar{p}_{i}}{z_{\alpha} \sigma_{i}}\right)^{2}-\frac{2}{\left(1^{T} G_{i}\right)^{2}} \sum_{\{j j ; j i\}} F_{i}^{T} C_{i j} F_{j}\right\} \ldots . .(7.13)
$$

Al igual que con la expresión (7.7), el cociente de Rayleigh conduce a:

$$
\theta_{\mathrm{i}} \leq \frac{1}{\lambda_{\max }}\left\{\left(\frac{\psi_{\mathrm{i}}-\overline{\mathrm{p}}_{\mathrm{i}}}{\mathrm{z}_{\alpha}}\right)^{2}-\frac{2}{\mathrm{~V}_{\mathrm{i}}^{2}} \sum_{\{\mathrm{j} j \mathrm{j} \neq \mathrm{i}\}} \mathrm{F}_{\mathrm{i}}^{\mathrm{T}} \mathrm{C}_{\mathrm{ij}} \mathrm{F}_{\mathrm{j}}\right\}
$$

Nótese que la diferencia con la relación (7.6), es que en (7.13) se hace un ajuste por la correlación que hay entre las probabilidades de impago de los créditos del segmento "i” con las de los créditos de los demás segmentos, representado por el término,

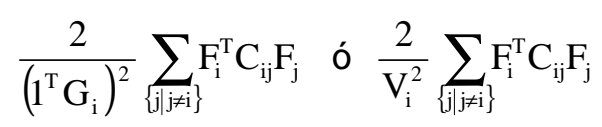

Evidentemente, cuando este término es positivo, como suele ser el caso, el efecto es el de reducir las cotas sobre los segmentos individuales. En resumen, se hace evidente como se obtienen límites individuales diferenciados por segmento, que dependen de tres factores, a saber:

1. La concentración de riesgo al interior del segmento, representada por el cociente $H\left(F_{i}\right) / H\left(G_{i}\right)$, o en su caso por el valor característico mayor de $\mathrm{M}_{\mathrm{i}}$.

2. La razón de suficiencia de capital idiosincrásica del segmento:

$$
\left(\frac{\psi_{i}-\bar{p}_{i}}{z_{\alpha} \sigma_{i}}\right)^{2}
$$

3. La corrección por correlación con otros segmentos:

$$
\frac{2}{\left(1^{\mathrm{T}} \mathrm{G}_{\mathrm{i}}\right)^{2}} \sum_{\{j \mathrm{j} ; \neq \mathrm{i}\}} \mathrm{F}_{\mathrm{i}}^{\mathrm{T}} \mathrm{C}_{\mathrm{ij}} \mathrm{F}_{\mathrm{j}} \quad \text { ó } \frac{2}{\mathrm{~V}_{\mathrm{i}}^{2}} \sum_{\{\mathrm{ij} j \mathrm{j} \mathrm{i}\}} \mathrm{F}_{\mathrm{i}}^{\mathrm{T}} \mathrm{C}_{\mathrm{ij}} \mathrm{F}_{\mathrm{j}}
$$




\subsubsection{Suficiencia de Capital para una Cartera Segmentada.}

$\mathrm{Al}$ integrar el análisis de los segmentos individuales a toda la cartera, es importante tomar en cuenta dos cosas. Primero, que los pesos relativos de cada segmento en la cartera no alteren los resultados obtenidos para la cartera no segmentada. Segundo, que se mantenga una propiedad de aditividad, que permita sumar los requerimientos de capital de los segmentos individuales, para obtener el requerimiento para la cartera en su conjunto. Esto puede lograrse de la siguiente manera:

Sea

$$
\phi=\frac{\sqrt{F^{T} M F}}{\sum_{i=1}^{h} \sqrt{F^{T} R_{i} F}}
$$

y defínase:

$$
v_{i}=\boldsymbol{\pi}_{\mathbf{i}}^{\mathbf{T}} \mathbf{F}_{i}+z_{\alpha} \phi \sqrt{\mathbf{F}^{T} \mathbf{R}_{i} \mathbf{F}} \leq K_{i}=\gamma_{i} K \text { para } \mathrm{i}=1,2, \ldots, \mathrm{h}
$$

Donde $\gamma_{i} \geq 0$ y $\sum_{i} \gamma_{i}=1$. Es fácil verificar que $\sum_{i} v_{i}=V a R_{\alpha}=\pi^{T} \mathbf{F}+z_{\alpha} \sqrt{\mathbf{F}^{\mathbf{T}} \mathbf{M} \mathbf{F}}$ Ahora, dividiendo (7.16) por $\mathrm{V}_{i}$, un análisis similar al de la sección anterior conduce a:

$$
\psi_{i} \geq \frac{v_{i}}{V_{i}}=\bar{p}_{i}+z_{\alpha} \phi \sigma_{i} \sqrt{H\left(G_{i}\right)+\frac{2}{\left(1^{T} G_{i}\right)^{2}} \sum_{\{j ; j \neq i} F_{i}{ }^{T} C_{i j} F_{j}}
$$

Resolviendo para $\mathrm{H}(\mathrm{Gi})$ se obtiene,

$$
\mathrm{H}\left(\mathbf{G}_{\mathrm{i}}\right) \leq\left(\frac{\psi_{\mathrm{i}}-\overline{\mathrm{p}}_{\mathrm{i}}}{\mathrm{z}_{\alpha} \phi \sigma_{\mathrm{i}}}\right)^{2}-\frac{2}{\left(\mathbf{1}^{\mathrm{T}} \mathrm{G}_{\mathrm{i}}\right)^{2}} \sum_{\{\mathrm{j} j \neq i \mathrm{i}\}} \mathrm{F}_{\mathrm{i}}^{\mathrm{T}} \mathrm{C}_{\mathrm{ij}} \mathrm{F}_{\mathrm{j}}
$$

y por el Teorema 6.1,

$$
\theta_{\mathrm{i}} \leq \frac{\mathrm{H}\left(\mathrm{F}_{\mathrm{i}}\right)}{\mathrm{H}\left(\mathrm{G}_{\mathrm{i}}\right)}\left\{\left(\frac{\psi_{\mathrm{i}}-\overline{\mathrm{p}}_{\mathrm{i}}}{\mathrm{z}_{\alpha} \phi \sigma_{\mathrm{i}}}\right)^{2}-\frac{2}{\left(1^{\mathrm{T}} \mathrm{G}_{\mathrm{i}}\right)^{2}} \sum_{\{\mathrm{j} j \mathrm{j} \neq \mathrm{i}\}} \mathrm{F}_{\mathrm{i}}^{\mathrm{T}} \mathrm{C}_{\mathrm{ij}} \mathrm{F}_{\mathrm{j}}\right\}
$$

En lo anterior, (7.17) establece la suficiencia de capital para los segmentos individuales, (7.18) establece un límite de concentración para el segmento y (7.19) provee la relación para los límites 
individuales. Usando el cociente de Rayleigh y los resultados del Teorema 6.1 pueden obtenerse las expresiones para todo lo anterior pero en términos de los valores característicos.

Nótese que (7.17) y las expresiones siguientes se obtienen de "vi/Vi", de manera que el peso de los segmentos en la cartera no se toma en cuenta. Por lo tanto, una simple suma de términos puede ser engañosa en cuanto a suficiencia de capital para la cartera segmentada. Por tanto, sea, $\gamma_{i}=\frac{V_{i}}{V}$. Entonces, por construcción, si (7.17) se satisface para todos los segmentos, $\psi=\sum_{i=1}^{h} \gamma_{i} \psi_{i}$ asegura la suficiencia de capital para la cartera.

Una vez más, el análisis nos lleva a unas relaciones simples a partir de las cuales se puede establecer la suficiencia de capital. Las expresiones obtenidas pueden usarse como instrumentos normativos para determinar límites individuales, cambios en la composición de la cartera y/o los ajustes al capital requeridos para mantener suficiencia si cambia el comportamiento de impago de los créditos en alguno o todos los segmentos.

\section{Ejemplo 7.2.}

Para ilustrar estos resultados, se usará la cartera mostrada en el Cuadro 6.1 de los ejemplos anteriores. Esta cartera se segmentó en tres grupos. El vector de créditos, las probabilidades de impago y la matriz de varianza-covarianza se partieron de acuerdo a:

$$
\mathrm{F}^{\mathrm{T}}=\left(\begin{array}{lll}
\mathrm{F}_{1} & \mathrm{~F}_{2} & \mathrm{~F}_{3}
\end{array}\right), \quad \pi^{\mathrm{T}}=\left(\begin{array}{lll}
\pi_{1} & \pi_{2} & \pi_{3}
\end{array}\right) \text { y } \quad \mathbf{M}=\left[\begin{array}{lll}
\mathbf{M}_{1} & \mathbf{C}_{1,2} & \mathbf{C}_{1,3} \\
\mathbf{C}_{2,1} & \mathbf{M}_{2} & \mathbf{C}_{2,3} \\
\mathbf{C}_{3,1} & \mathbf{C}_{3,2} & \mathbf{M}_{3}
\end{array}\right] \text { donde: }
$$

- $\mathrm{M}_{1}$, es la matriz de varianza-covarianza idiosincrática del primer grupo, que en este ejemplo consiste de ocho créditos: A1, C2, C4, D1, D3, F1, $\mathrm{F} 4$ y G2. ${ }^{26}$

- $\mathrm{M}_{2}$, es la matriz de varianza-covarianza idiosincrática del segundo grupo. En este ejemplo está formado por los ocho créditos siguientes: B1, C1, C3, E2, E3, F3, G1 y G5.

\footnotetext{
${ }^{26} \mathrm{~A} 1$ es el primer crédito calificado con $A, \mathrm{C} 2$ es el segundo crédito calificado con $C$ y así sucesivamente.
} 
- $\mathrm{M}_{3}$, es la matriz de varianza-covarianza idiosincrática del tercer grupo, que en este ejemplo se conforma de los nueve créditos restantes: A2, B2, C5, D2, E1, F2, G3, G4 y G6.

- $\mathrm{C}_{12}{ }^{\mathrm{T}}=\mathrm{C}_{21}$, es la matriz de covarianzas entre los créditos de los grupos uno y dos. Análogamente, $\mathrm{C}_{13}{ }^{\mathrm{T}}=\mathrm{C}_{31}$ es la matriz de covarianzas entre los créditos del primer y segundo grupos y $\mathrm{C}_{23}{ }^{\mathrm{T}}=\mathrm{C}_{32}$ es la matriz de covarianzas entre los créditos del segundo y tercer grupos.

Para aplicar los resultados, el primer paso es calcular el valor de cada segmento, su correspondiente índice de Herfindahl Hirschman, y su asignación de capital. Estos números se resumen en el siguiente cuadro:

\section{Cuadro 7.3.}

\begin{tabular}{|c|c|c|c|c|}
\hline Segmento $\mathbf{i}$ & $\mathbf{V}_{\mathbf{i}}$ & $\mathbf{H}\left(\mathbf{F}_{\mathbf{i}}\right)$ & $\boldsymbol{\gamma}_{\mathbf{i}}$ & $\mathbf{K}_{\mathbf{i}}$ \\
\hline $\mathbf{1}$ & $\$ 44,024$ & 0.2613 & 0.3382 & $\$ 20,293$ \\
\hline $\mathbf{2}$ & $\$ 43,186$ & 0.2008 & 0.3318 & $\$ 19,907$ \\
\hline $\mathbf{3}$ & $\$ 42,954$ & 0.1293 & 0.3300 & $\$ 19,800$ \\
\hline
\end{tabular}

Nótese que para cada segmento, la forma de la matriz $\mathbf{R}_{\mathrm{i}}$ es:

$$
\mathbf{R}_{1}=\left[\begin{array}{ccc}
M_{1} & C_{12} & C_{13} \\
C_{21} & 0 & 0 \\
C_{31} & 0 & 0
\end{array}\right], \mathbf{R}_{2}=\left[\begin{array}{ccc}
0 & C_{12} & 0 \\
C_{21} & M_{2} & C_{23} \\
0 & C_{32} & 0
\end{array}\right], y \mathbf{R}_{3}=\left[\begin{array}{ccc}
0 & 0 & C_{13} \\
0 & 0 & C_{23} \\
C_{31} & C_{32} & M_{3}
\end{array}\right]
$$

De (7.15), el parámetro $\phi$ que permite la suma de los VaR ajustados es:

$$
\phi=\frac{\sqrt{\mathrm{F}^{\mathrm{T}} \mathrm{MF}}}{\sum_{\mathrm{i}} \sqrt{\mathrm{F}^{\mathrm{T}} \mathrm{R}_{\mathrm{i}} \mathrm{F}}}=0.4622
$$

Al calcular $v_{i}$ con (7.16), usando un nivel de confianza de 5\% y suponiendo normalidad se obtienen las siguientes relaciones para suficiencia de capital: $v_{l}=\$ 16,255<\mathrm{K}_{1}=\$ 20,293, v_{2}=\$ 19,368$ $<\mathrm{K}_{2}=\$ 19,907, v_{3}=\$ 20,060>\mathrm{K}_{3}=\$ 19,800$. Nótese que el capital asignado al último segmento no cubre su riesgo. De hecho, es el segmento más riesgoso de los tres. Esto significa que este último grupo tampoco satisface las demás condiciones. 
Siguiendo con el ejemplo, la matriz de varianza-covarianza idiosincrática $\mathrm{M}_{\mathrm{i}}$ para cada grupo se factoriza para obtener $S_{i}$, y con ello se obtienen los vectores correspondientes redimensionados $G_{i}$. En el siguiente cuadro se resumen los resultados:

Cuadro 7.4

\begin{tabular}{|c|c|c|c|c|}
\hline & & $F_{1}$ & & $\mathrm{G}_{1}$ \\
\hline $\mathrm{A} 1$ & & 4,728 & & 1,043 \\
\hline C2 & & 3,204 & & 1,362 \\
\hline C4 & & 4,912 & & 1,699 \\
\hline D1 & & 5,320 & & 2,092 \\
\hline D3 & & 20,239 & & 5,651 \\
\hline $\mathrm{F} 1$ & & 1,933 & & 1,608 \\
\hline F4 & & 2,598 & & 1,823 \\
\hline G2 & & 1,090 & & 1,571 \\
\hline Total= & $\$$ & 44,024 & $\$$ & 16,849 \\
\hline
\end{tabular}

\begin{tabular}{|c|c|c|c|c|}
\hline & & $F_{2}$ & & $\mathrm{G}_{2}$ \\
\hline B1 & & 5,528 & & 1,603 \\
\hline C1 & & 3,138 & & 1,529 \\
\hline C3 & & 4,831 & & 1,853 \\
\hline E2 & & 5,042 & & 2,481 \\
\hline E3 & & 15,411 & & 5,211 \\
\hline F3 & & 2,411 & & 2,042 \\
\hline G1 & & 358 & & 1,630 \\
\hline G5 & & 6,467 & & 4,087 \\
\hline Total $=$ & $\$$ & 43,186 & $\$$ & 20,436 \\
\hline
\end{tabular}

\begin{tabular}{|c|c|c|c|}
\hline & & $F_{3}$ & $\mathrm{G}_{3}$ \\
\hline A2 & & 7,728 & 1,637 \\
\hline B2 & & 5,848 & 1,895 \\
\hline C5 & & 5,435 & 2,284 \\
\hline D2 & & 5,765 & 2,756 \\
\hline E1 & & 1,800 & 2,181 \\
\hline $\mathrm{F} 2$ & & 2,317 & 2,634 \\
\hline G3 & & 2,652 & 3,298 \\
\hline G4 & & 4,929 & 4,086 \\
\hline G6 & & 6,480 & 4,622 \\
\hline Total= & $\$$ & 42,954 & 25,392 \\
\hline
\end{tabular}

Es interesante notar que las carteras redimensionadas se reducen en valor, y no sólo eso sino que los valores son más uniformes, como se aprecia en los índices de Herfindahl. Además, como $\gamma_{\mathrm{i}}=\frac{\mathrm{V}_{\mathrm{i}}}{\mathrm{V}}$, necesariamente se tiene $\psi_{i}=\frac{K_{i}}{V_{i}}=\frac{\gamma_{i} \times K}{V_{i}}=\frac{K}{V}=\frac{60,000}{130,164}=0.4610$ para todos los segmentos. El siguiente cuadro muestra los índices de concentración, las probabilidades esperadas de impago y las $\sigma_{i}$ obtenidas:

\section{Cuadro 7.5}

\begin{tabular}{|c|c|c|c|c|}
\hline Segmento $\mathbf{i}$ & $\mathbf{H}\left(\mathbf{G}_{\mathbf{i}}\right)$ & $\boldsymbol{\psi}_{\mathbf{i}}$ & $\overline{\mathbf{p}_{\mathbf{i}}}$ & $\boldsymbol{\sigma}_{\mathbf{i}}$ \\
\hline $\mathbf{1}$ & 0.1779 & 0.4610 & 0.0774 & 0.3827 \\
\hline $\mathbf{2}$ & 0.1561 & 0.4610 & 0.1162 & 0.4732 \\
\hline $\mathbf{3}$ & 0.1236 & 0.4610 & 0.1339 & 0.5912 \\
\hline
\end{tabular}

Con estos valores, pueden verificarse las relaciones (7.18) y (7.19) para todos los segmentos de la cartera. Como era de esperarse, el tercer grupo incumple (7.18):

$$
\mathrm{H}\left(\mathbf{G}_{3}\right)=0.1236>\left(\frac{\psi_{\mathrm{i}}-\overline{\mathrm{p}}_{\mathrm{i}}}{\mathrm{z}_{\alpha} \phi \sigma_{\mathrm{i}}}\right)^{2}-\frac{2}{\left(\mathbf{1}^{\mathrm{T}} \mathrm{G}_{\mathrm{i}}\right)^{2}} \sum_{\{\mathrm{i} j \neq \mathrm{j}\}} \mathrm{F}_{\mathrm{i}}^{\mathrm{T}} \mathrm{C}_{\mathrm{ij}} \mathrm{F}_{\mathrm{j}}=0.1097
$$

Ahora, de (7.19) se obtienen los límites suficientes para los créditos de cada grupo $\left(f_{1}, f_{2}, f_{3}\right)$, que implican suficiencia de capital:

$$
\theta_{1} \leq \frac{0.2613}{0.1779} \times 0.6933=1.0179 ; f_{1} \leq 1.0179 \times \$ 44,024=\$ 44,812
$$




$$
\begin{aligned}
& \theta_{2} \leq \frac{0.2008}{0.1561} \times 0.2020=0.2598 ; f_{2} \leq 0.2598 \times \$ 43,186=\$ 11,222 \\
& \theta_{3} \leq \frac{0.1293}{0.1236} \times 0.1097=0.1148 ; f_{3} \leq 0.1148 \times \$ 42,954=\$ 4,930
\end{aligned}
$$

Evidentemente, dado que la cota para los créditos del primer grupo es $\theta_{1}=1.018>1$ todos los créditos del grupo están dentro del límite. En el segundo grupo, el crédito de \$15,411 excede el límite. Finalmente, como era de esperarse el tercer grupo es el más problemático ya que sólo los cuatro créditos más pequeños respetan el límite.

Es interesante analizar los efectos de la correlación y el procedimiento de redimensionamiento en este ejemplo. Lo anterior establece que el primer grupo puede tener créditos de cualquier tamaño, pero por otro lado, el segmento más riesgoso debe incluir sólo créditos muy pequeños, lo cual aunque es lógico, claramente no se cumple.

Ahora, con respecto a la suficiencia de capital, se examina (7.17) y al comparar $\psi \operatorname{con} \sum \gamma_{i} \frac{v_{i}}{V_{i}}$, se obtiene:

$$
\psi=0.4610 \geq \sum_{\mathrm{i}=1}^{3} \frac{v_{\mathrm{i}}}{\mathrm{V}_{\mathrm{i}}}=\frac{\mathrm{VaR}_{\alpha}}{\mathrm{V}}=\frac{55,684}{130,164}=0.4278
$$

Nótese que el resultado es el mismo que el del ejemplo 7.1, con lo cual se verifica que la cartera cuenta con suficiencia de capital, a pesar de que el tercer segmento en lo individual, viola todos los requisitos. Es interesante notar el poder analítico de la herramienta aquí desarrollada, dado que si se restringe el ejercicio al uso del modelo general como en el ejemplo 7.1, sin analizar los segmentos individuales, el tercer segmento que es claramente un grupo riesgoso de créditos, hubiera pasado desapercibido. Finalmente, es además evidente, que los resultados dependen del criterio de segmentación usado, dado que se pueden clasificar los créditos de manera que todos los segmentos cumplan con las relaciones que sean relevantes, en cuyo caso los grupos riesgosos no se detectarían. Sin embargo, el ejemplo indica claramente como obtener una idea de la concentración ex ante, en el peor de los casos por prueba y error. Un poco más de investigación seguramente conducirá a una búsqueda más sistemática. 


\subsection{La Inclusión de Tasas de Recuperación.}

Es sencillo ampliar todas las relaciones obtenidas hasta ahora, para tomar en cuenta de forma explícita las tasas de recuperación de los créditos. Hacerlo, conduce a límites menos estrictos en términos de la concentración tolerable en los distintos segmentos. Básicamente, existen dos maneras de incluir a las tasas de recuperación en el análisis. La primera es definir $\mathbf{F}$ directamente como el vector de "pérdidas dado incumplimiento" (PDI) en lugar del saldo pendiente, donde se supone que nada se recupera si los deudores no pagan. Esto estaría más de acuerdo con la práctica actual2 ${ }^{27}$. Entonces, si se tiene una estimación del vector PDI, puede usarse directamente en las relaciones antes obtenidas sin cambio alguno, pero deben interpretarse correctamente.

Explícitamente, incluir las tasas de recuperación implica hacer una transformación inicial del vector original de créditos. Sea " $\boldsymbol{\Delta}$ " la matriz diagonal, cuyos elementos distintos de cero son las tasas de pérdida dado incumplimiento. El vector de pérdidas dado incumplimiento para la cartera es entonces: $\boldsymbol{P}=\boldsymbol{\Delta} \boldsymbol{F}$ y sea $\boldsymbol{V}{ }^{\prime}=\boldsymbol{I}^{\boldsymbol{T}} \boldsymbol{P}$. Para incluir una correlación, se calcula la transformación $\boldsymbol{G}=\boldsymbol{S P}$. Así, el vector original se modifica de hecho dos veces i.e. $\boldsymbol{G}=\boldsymbol{S} \boldsymbol{\Delta} \boldsymbol{F}$. Con un poco de álgebra se llega a las relaciones de suficiencia de capital, concentración y límites individuales, las cuales muestran como los cambios en la matriz de pérdida dado incumplimiento " $\Delta$ ", afectan los parámetros.

Aunque la transformación sugerida conduce a un modelo completamente general, es difícil visualizar el impacto de cambiar las tasas de recuperación en el riesgo de crédito, la concentración y la suficiencia de capital. Además, debe tomarse en cuenta que las tasas de recuperación son por sí mismas variables aleatorias, de manera que esta matriz puede variar de acuerdo a las condiciones económicas y de mercado. Por ello, se desearía poder explorar fácilmente la sensibilidad de las relaciones de suficiencia de capital, concentración y límites individuales a cambios en las tasas de recuperación. Entonces, considérese la alternativa de suponer, por ejemplo ${ }^{28}$, que la cartera está segmentada de manera que las tasas de recuperación sean las mismas para todos los créditos del grupo. Bajo la estructura de la sección anterior, sea " $r_{\mathbf{i}}$ " la tasa de recuperación de los créditos que caigan en impago en el segmento "i", de manera que la pérdida dado incumplimiento es simplemente $\mathbf{P}_{\mathbf{i}}=\left(1-r_{i}\right) \mathbf{F}_{\mathbf{i}}$. Procediendo de la manera usual:

\footnotetext{
${ }^{27}$ Véase el documento de "Credit Risk Modeling" del Comité de Basilea de Supervisión Bancaria. Abril 1999.

${ }^{28}$ Esto es lo que hace CreditRisk ${ }^{+}$.
} 
$\sum_{i}\left(1-r_{i}\right) \lambda_{i}^{2} H\left(\mathbf{G}_{i}\right)+\frac{2}{\left(\mathbf{1}^{\mathrm{T}} \mathbf{G}\right)^{2}} \sum_{\mathrm{i}}\left(1-\mathrm{r}_{\mathrm{i}}\right) \sum_{\{j \mathrm{j} \neq \mathrm{i}\}}\left(1-\mathrm{r}_{\mathrm{j}}\right) \mathrm{F}_{\mathrm{i}}^{\mathrm{T}} \mathrm{C}_{\mathrm{ij}} \mathrm{F}_{\mathrm{j}} \leq\left(\frac{\psi-\frac{1}{\mathrm{~V}} \sum_{\mathrm{i}}\left(1-\mathrm{r}_{\mathrm{i}}\right) \pi_{\mathrm{i}}^{\mathrm{T}} \mathrm{F}_{\mathrm{i}}}{\mathrm{z}_{\alpha} \sigma}\right)^{2} \ldots$

Esta expresión muestra claramente que cualquier cambio en las tasas de recuperación tiene un impacto doble. Por un lado, un aumento (disminución) en la tasa de recuperación de cualquier segmento en particular, reduce (incrementa) la importancia de la contribución de la concentración de estos segmentos (parte izquierda de la desigualdad). Adicionalmente, su contribución a la pérdida esperada también disminuye (aumenta) el numerador del lado derecho, incrementando (disminuyendo) la cota establecida para la concentración. Además, no es difícil probar que el denominador del lado derecho de la desigualdad, se comporta de la manera esperada: disminuyendo al aumentar la tasa de recuperación y viceversa.

Finalmente, es necesario señalar que no se requiere hacer la segmentación por pérdidas iguales dado incumplimiento. Aunque esto fue conveniente para hacer evidente el impacto de las tasas de recuperación sobre la concentración, los límites y los requisitos de capital, se puede adecuar el modelo haciendo la transformación del vector de saldos, y conservar la flexibilidad del modelo para segmentar la cartera de cualquier manera que se estime conveniente y realizar el análisis correspondiente para detectar segmentos riesgosos.

\section{El Supuesto de Normalidad y una Visión Sistémica.}

Hasta el momento, en todos los ejemplos se ha considerado que la distribución de pérdidas se aproxima con una distribución Normal. En esta sección se discutirá la posibilidad de aproximar dicha distribución con otras que quedan totalmente caracterizadas por la media y la varianza, en particular, la distribución Gama. La distribución Gama es conveniente porque, además de que queda totalmente especificada por su media y su varianza, puede captar la asimetría que típicamente presentan las distribuciones de pérdidas atribuibles a carteras de créditos. Una forma de representar la función de densidad Gama es la siguiente ${ }^{29}$ :

$$
f(x \mid \alpha, \beta)=\frac{x^{\alpha-1}}{\beta^{\alpha} \Gamma(\alpha)} e^{-\frac{x}{\beta}}
$$

\footnotetext{
${ }^{29}$ Hay muchas formas de representar la distribución Gama. La parametrización presentada es muy usual y es la que se utiliza en el desarrollo de CreditRisk ${ }^{+}$, según se aprecia en el manual.
} 
La media y la varianza de la distribución Gama son, $E(x)=\alpha \beta \quad y \quad V A R(x)=\alpha \beta^{2}$, respectivamente.

Se ha realizado una serie de ejercicios comparativos, entre el modelo desarrollado y CreditRisk ${ }^{+}$. Sin pretender que la investigación es exhaustiva ni rigurosamente científica, los resultados obtenidos hasta el momento son alentadores. En el ejemplo siguiente se presentan las distribuciones de pérdidas obtenidas mediante CreditRisk $^{+}$y CyRCE para dos carteras elegidas de manera aleatoria.

\section{Ejemplo 8.1.}

El primer ejercicio muestra la distribución de pérdidas para una cartera aleatoria de 3000 créditos tomados del SENICREB ${ }^{30}$. Como puede apreciarse en este ejemplo, si bien con la aproximación normal, el VaR difiere hasta en un $37.7 \%$ con respecto a la cifra que produce CreditRisk+, en el caso de la aproximación a través de la distribución Gama, la diferencia es solamente del $0.45 \%$. A nuestro juicio, esta diferencia no es significativa ni justifica el esfuerzo computacional adicional requerido para aplicar CreditRisk+.

\section{GRÁFICA 8.1}

\section{Distribuciones de Probabilidad de Pérdidas Obtenidas Mediante CreditRisk + y CyRCE, haciendo el Ajuste Mediante la Gama y la Normal para una Muestra Aleatoria de 3000 Créditos}

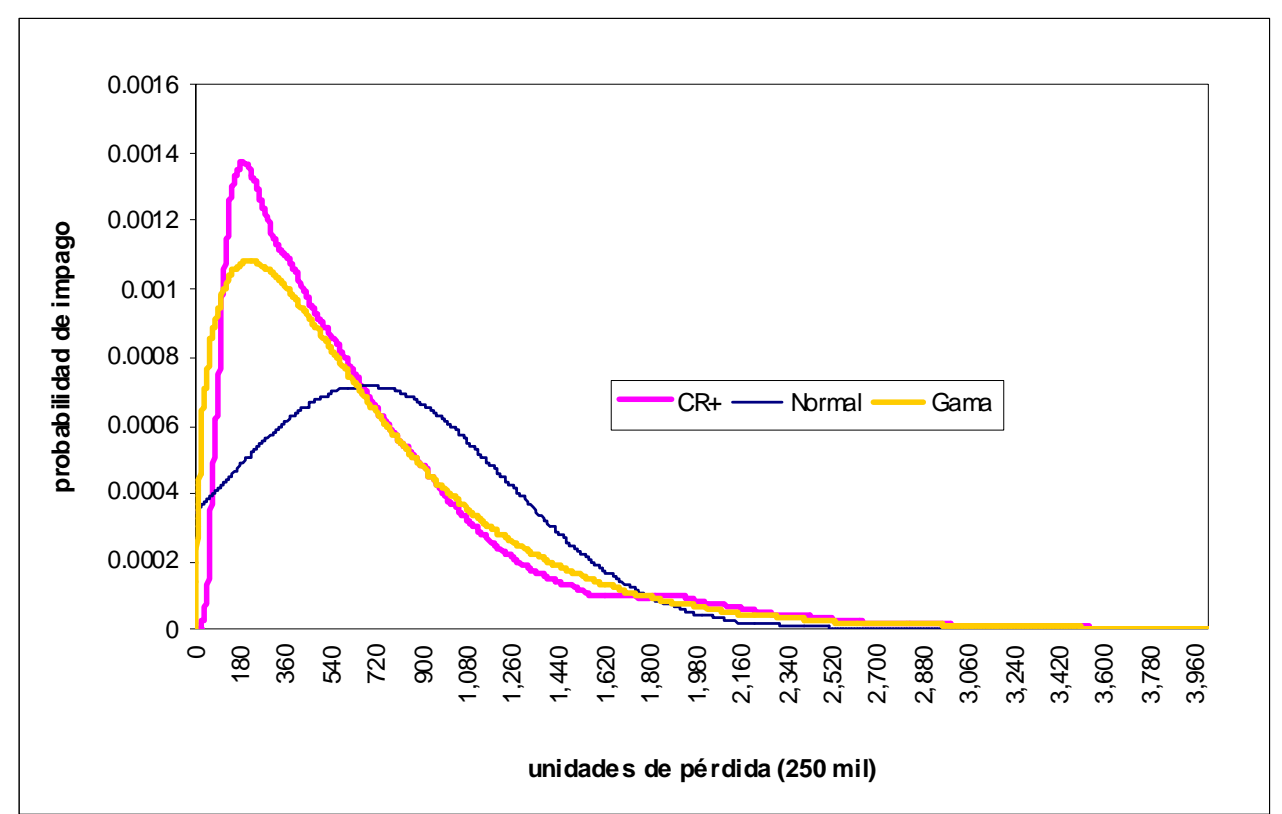

\footnotetext{
${ }^{30}$ SENICREB (Servicio Nacional de Información de Créditos Bancarios), es una base de datos administrada
} por el Banco de México que contiene todos los créditos del sistema bancario. 
Cuadro 8.1: Estadísticas de las Distribuciones de Pérdidas Obtenidas.

\begin{tabular}{|l|c|c|c|c|}
\hline VaR en unidades de pérdida & $\mathbf{0 . 9 5}$ & $\mathbf{0 . 9 7 5}$ & $\mathbf{0 . 9 9}$ & $\mathbf{0 . 9 9 5}$ \\
\hline CR+ & 1,878 & 2,212 & 2,623 & 2,932 \\
\hline Normal & 1,590 & 1,765 & 1,969 & 2,108 \\
\hline Gama & 1,770 & 2,120 & 2,577 & 2,919 \\
\hline
\end{tabular}

\begin{tabular}{|l|c|c|c|c|c|}
\hline Estadísticas en unidades & E(x) & VAR(x) & Desv. Estándar(x) & alfa & beta \\
\hline CR+ & 673 & 312,277 & 559 & - & - \\
\hline Normal & 674 & 310,116 & 557 & - & - \\
\hline Gama & 674 & 310,116 & 557 & 1.46 & 460.27 \\
\hline
\end{tabular}

El segundo ejemplo repite el ejercicio para una cartera de 1320 créditos tomados de manera aleatoria del SENICREB. Nótese que la distribución de pérdidas obtenida mediante la aplicación de CreditRisk $^{+}$es bi-modal. Esto se debe a que la cartera contenía un crédito muy grande, en relación con los demás de la muestra, y al requerimiento de CreditRisk $^{+}$de agrupar los créditos en "cubetas" de unidades de pérdida homogéneas, que genera una discontinuidad en las pérdidas posibles, al quedar varias cubetas vacías. Como se aprecia en la Gráfica 8.2 y en el cuadro adjunto, haciendo un ajuste con la distribución Gama para CyRCE, la mayor discrepancia se presenta en el VaR al 99\% de confianza, con respecto al que se obtiene utilizando CreditRisk ${ }^{+}$y es de $12.34 \%$. De los ejercicios realizados a la fecha, este es uno de los peores resultados.

GRÁFICA 8.2

\section{Distribuciones de Probabilidad de Pérdidas Obtenidas Mediante CreditRisk+} y CyRCE, Haciendo el Ajuste Mediante la Gama y la Normal para una Muestra Aleatoria de 1320 Créditos

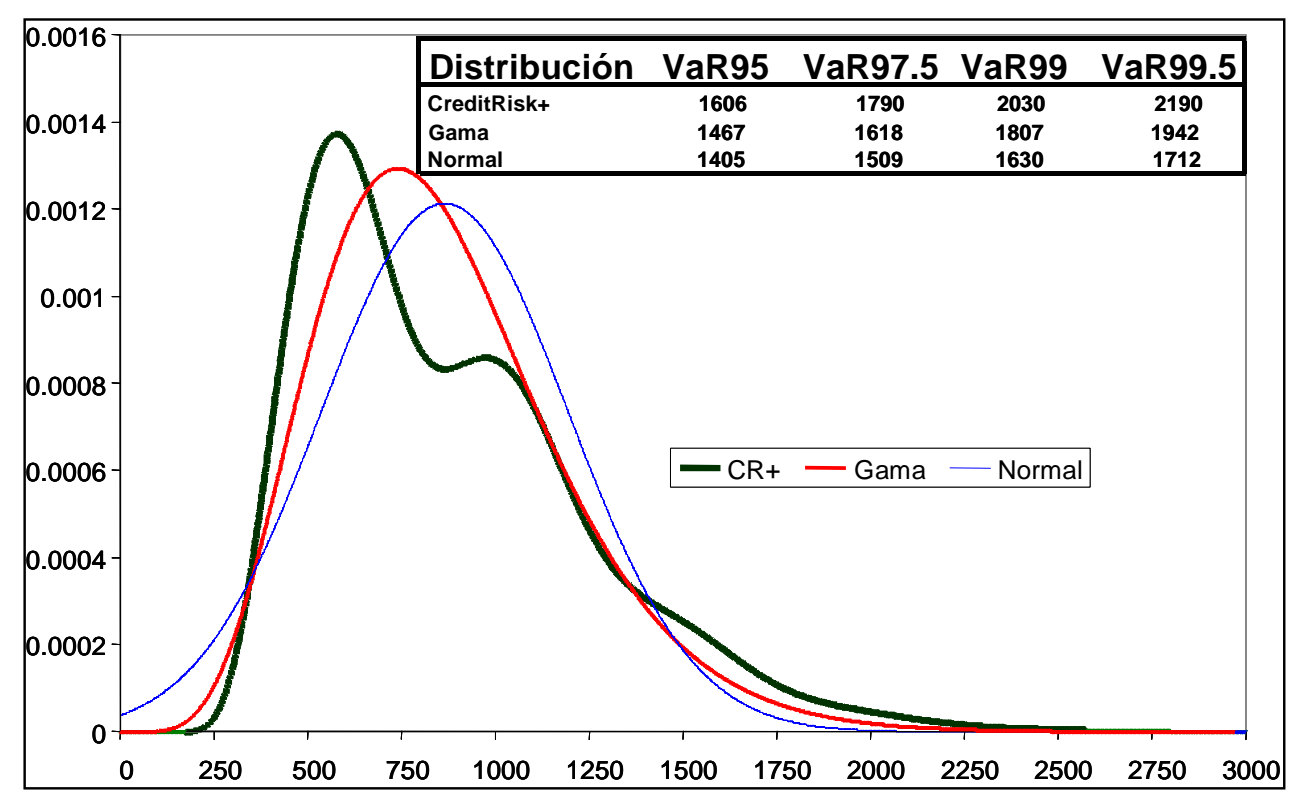


Finalmente se señala que no siempre es el caso que el VaR obtenido por CyRCE, subestima el que se consigue mediante CreditRisk ${ }^{+}$. Se han realizado ejercicios donde sucede lo contrario, y normalmente en esos casos, las diferencias en términos porcentuales son aún más pequeñas que en el primer ejemplo mostrado.

Dada la eficiencia de cálculo que se logra utilizando CyRCE, es posible analizar todo el sistema bancario y obtener algunas estadísticas interesantes. Esto se ilustra en el ejemplo siguiente.

\section{Ejemplo 8.2.}

En este ejemplo se presenta el análisis de riesgo de crédito para los 20 bancos que más contribuyen al riesgo del sistema financiero mexicano. En este ejercicio se utilizaron los datos del SENICREB. Los nombres de los bancos se omiten por razones de confidencialidad. En la Gráfica 8.3, se muestra un histograma que representa la concentración del crédito en los bancos, medida con el índice de Herfindahl. De este ejercicio se obtiene que la concentración del crédito en los bancos mexicanos es de 0.0163 en promedio.

\section{Gráfica 8.3}

\section{Concentración de la Cartera de la Banca Mexicana Medida con el Î́ndice de Herfindahl-Hirschman}

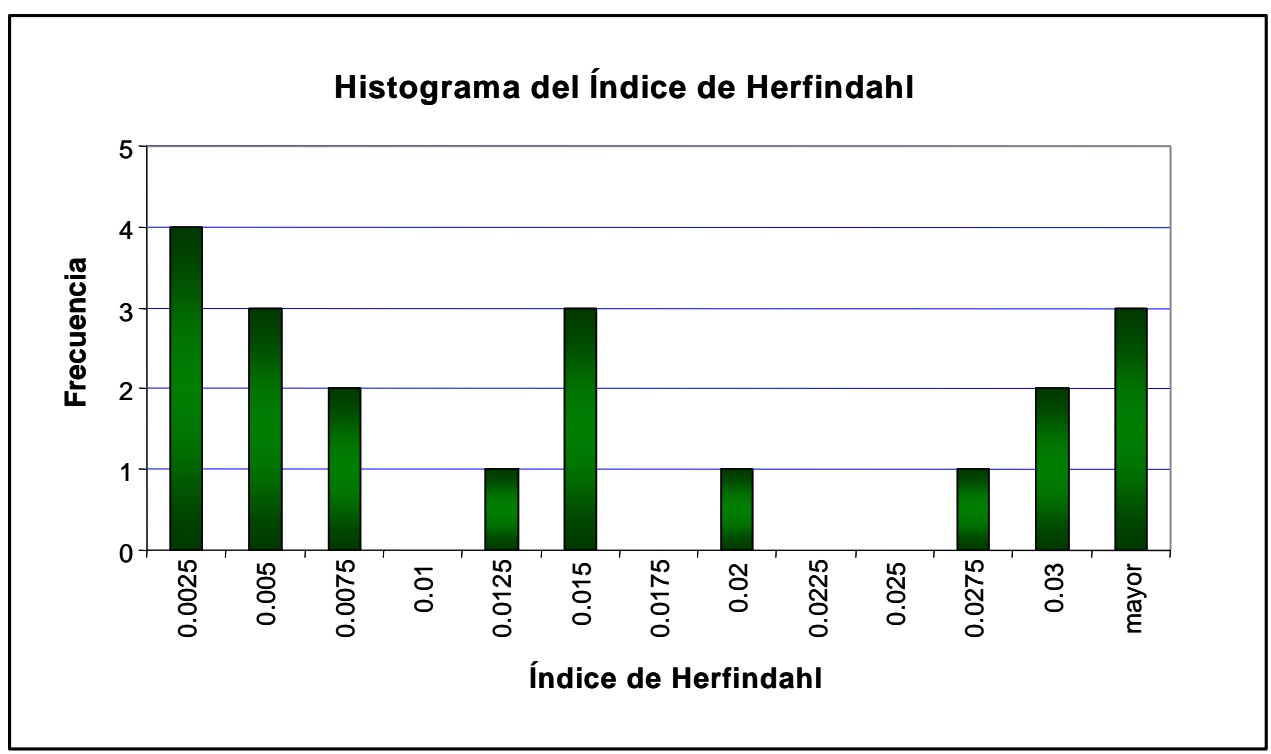

En la Gráfica 8.4, se aprecia como está distribuida la probabilidad de incumplimiento de los bancos del sistema, mientras que en la Gráfica 8.5, se observa el cumplimiento de suficiencia de capital. Así, para el ejercicio presentado, la probabilidad de impago promedio en el sistema es de 0.0813 . 
Nótese que si se toma el capital contable como capital económico, solamente hay dos bancos que están en una región sensible de incumplimiento, ya que la relación de VaR sobre Capital contable para esos bancos es superior a $75 \%$. En promedio, $\mathrm{VaR} / \mathrm{CC}=38 \%$ para el sistema.

\section{Gráfica 8.4}

Histograma de la Distribución de la Probabilidad de Incumplimiento de la Banca Mexicana

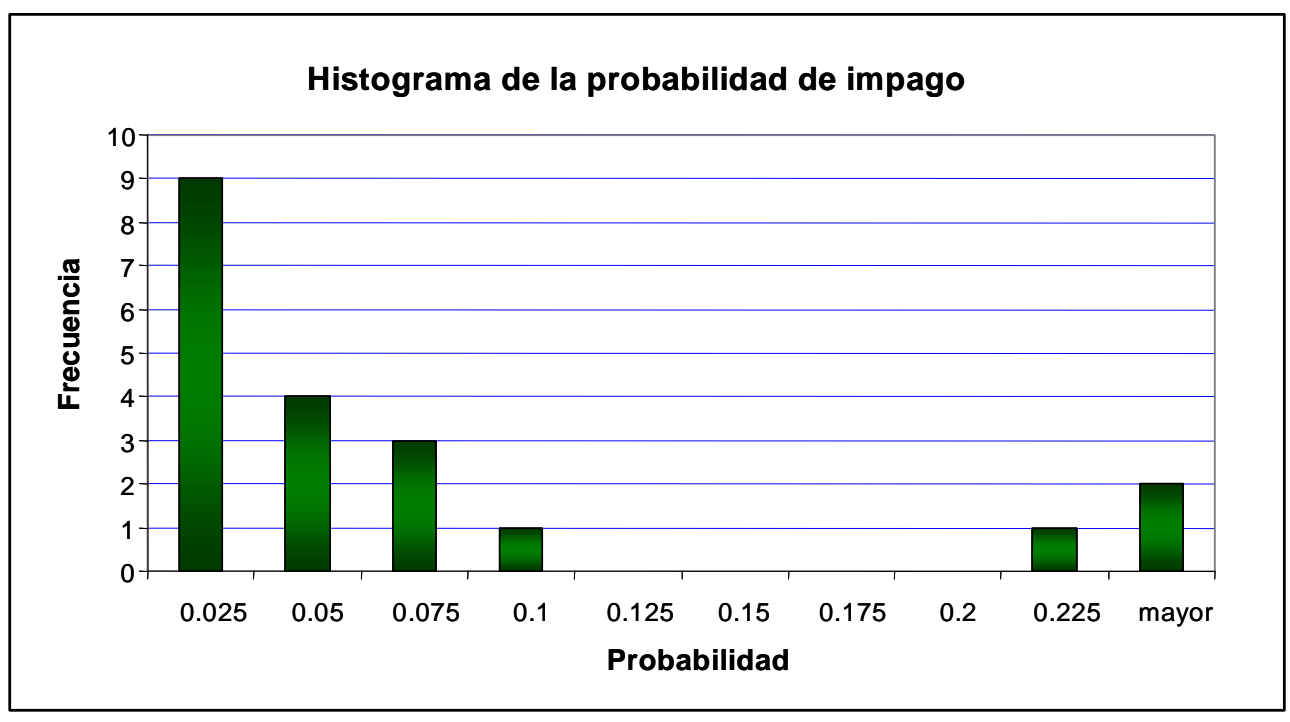

Gráfica 8.5

Histograma del Cumplimiento del Requerimiento de Capital según CyRCE para la Banca Mexicana

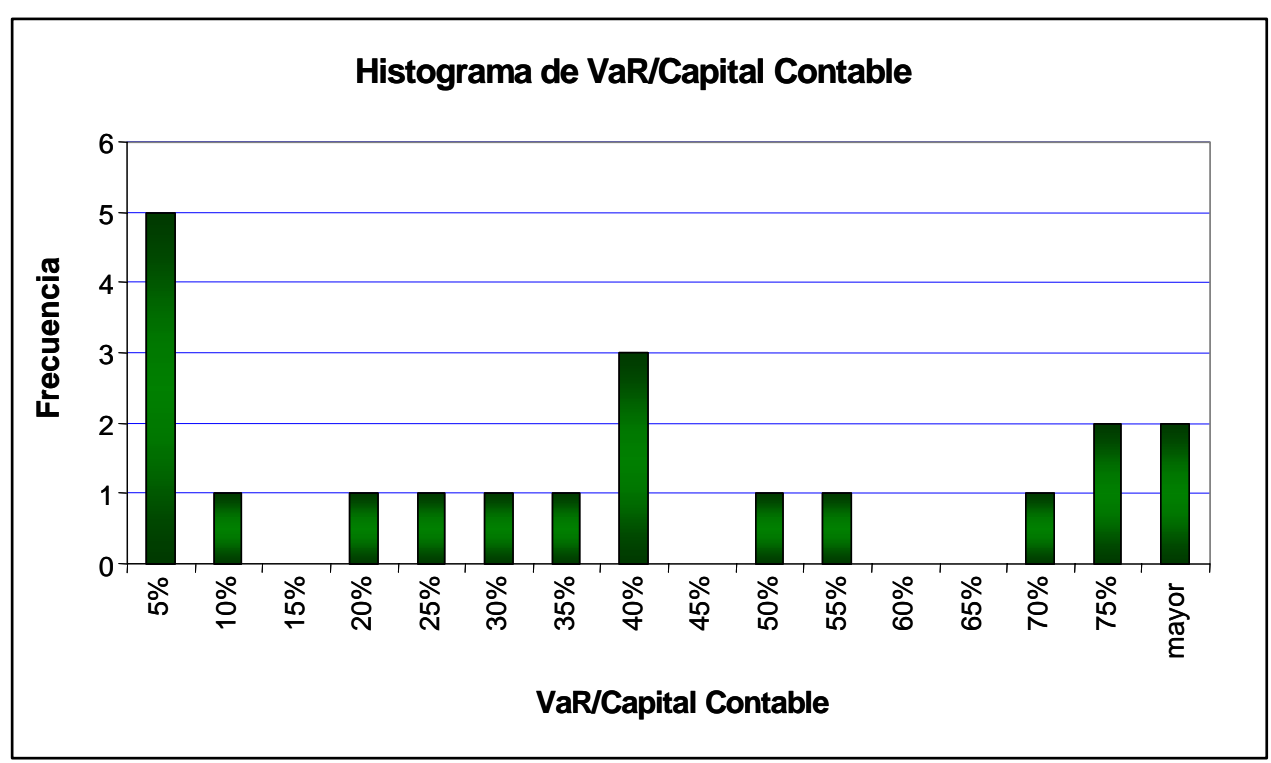


Finalmente, en la Gráfica 8.6, se presenta la forma en que está concentrado el riesgo crediticio del sistema bancario mexicano. En ella se aprecia que más de $96 \%$ del VaR del sistema está concentrado en seis bancos.

Gráfica 8.6
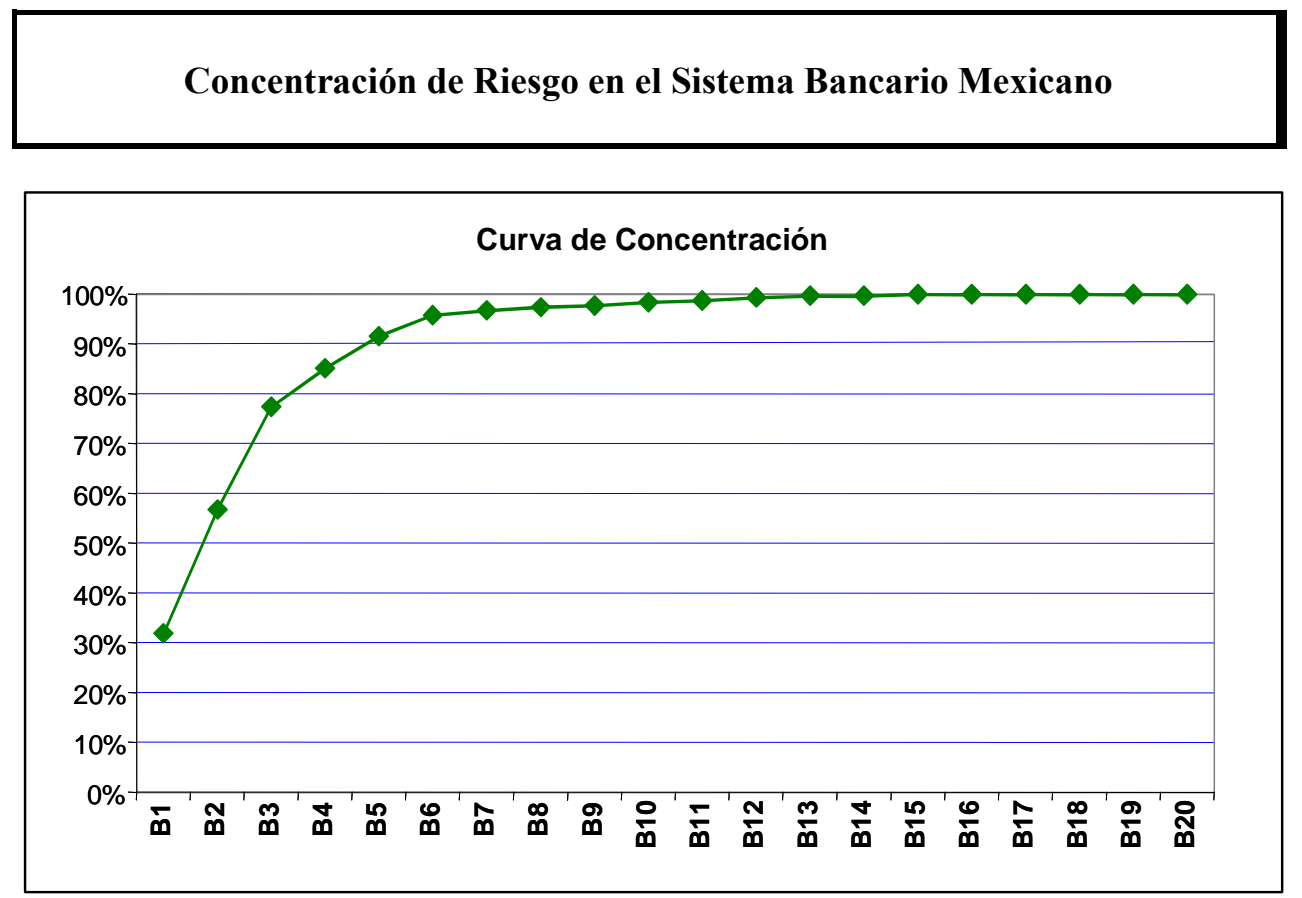

Los ejercicios anteriores son solamente una muestra del tipo de análisis que permite la metodología.

\section{Conclusiones}

Los resultados obtenidos hasta ahora son muy convenientes para administrar el riesgo de crédito, ya que proporcionan fórmulas explícitas para medir el riesgo y permiten una cuantificación precisa de las acciones normativas que deben tomarse para mantener la suficiencia de capital. Además, el modelo proporciona medios simples para determinar los efectos de la correlación y la concentración en el riesgo de la cartera de créditos. Dado que los límites individuales y el índice de HerfindahlHirschman están relacionados con la concentración, y las medidas están sujetas a la misma cota, cualquier indicador puede usarse como instrumento normativo. Además, no hay razón para no usar ambas medidas de manera conjunta. Por ejemplo, si un ejecutivo de cuenta decide otorgar un crédito haciendo caso omiso del límite individual establecido por norma, la gravedad de la infracción puede evaluarse mediante el índice de Herfindahl. Puede ser que la infracción a los límites no sea seria en términos de incremento del riesgo. Finalmente, si las tasas de recuperación y 
de impago cambian, o la concentración en una dimensión en particular parece excesiva, las relaciones pueden usarse para determinar los ajustes a la razón de capitalización y/o a la composición de la concentración de la cartera para reestablecer la suficiencia de capital. Obviamente, el grado en el que los bancos tengan control sobre las tasas de recuperación e impago, haciendo eficiente su gestión en materia de cobranza y recuperación y haciendo uso de derivados de crédito, puede ser parte de la mezcla de instrumentos de administración que pueden usarse para mantener la suficiencia de capital.

Claramente, se recomienda usar el índice de concentración y los límites de manera conjunta, ya que ambos tienen sus méritos y sus problemas. Mientras que el límite individual es fácil de instrumentar y supervisar, puede conducir a restringir demasiado las distribuciones de los créditos. Por otro lado, aunque el índice de Herfindahl permite más flexibilidad en la configuración de la cartera de crédito, es mucho más difícil de instrumentar, y las logísticas pueden resultar difíciles de manejar. Para ilustrar este punto, considérese el caso simple de concentración en sólo una dimensión de créditos no correlacionados con probabilidades iguales de impago. Supóngase que la administración está considerando otorgar un nuevo crédito. Aún para este caso simple de una dimensión, se debe comprobar que no se viole la condición de la suficiencia de capital. Mientras que en la regla individual para el crédito sólo es necesario verificar que el tamaño del crédito no exceda el límite establecido, aún suponiendo independencia, el uso del índice de Herfindahl implicaría verificar que se mantenga la desigualdad $\frac{1}{V}|F| \leq \sqrt{\theta}$. Si sólo un crédito " $f$ " está incluido, la condición que debe satisfacerse para no violar la desigualdad es:

$$
\|F\|^{2}+f^{2} \leq \theta(V+f)^{2}
$$

Esto significa que debe resolverse una ecuación de segundo grado para llegar a:

$$
f \leq 2 \theta+V \sqrt{(1-\theta)\left[\theta-\frac{\|\left.\right|^{2}}{V^{2}}\right]}
$$

Aunque manejable, esto es claramente mucho más complicado que la regla tradicional. Ahora imagínese la situación de un comité tradicional de crédito, donde se está considerando la aprobación simultánea de muchos créditos, y éstos pueden estar correlacionados en distintos segmentos: la logística puede probar ser un reto formidable. 
Esto se debe a los resultados obtenidos donde se esclarece que la concentración en números no necesariamente es un indicador de riesgo, pero sí se debe ver cómo qué está concentrado el riesgo mediante la transformación $\mathbf{G}=\mathbf{S F}$.

Finalmente, es evidente que las distribuciones de la probabilidad de impago así como las tasas de recuperación, muestran comportamientos aleatorios en el tiempo, dependiendo de varios factores económicos y financieros. Contrario al riesgo de mercado, donde los factores de riesgo pueden modelarse usando procesos continuos, porque los incumplimientos de los créditos son eventos discretos en el tiempo, el comportamiento de impago en un grupo en particular puede cambiar también en saltos discretos pronunciados. En particular el punto de vista del autor es que precisamente por eso es difícil establecer una concentración ex-ante, a saber: bajo diferentes condiciones económicas y crediticias, las probabilidades de impago y las correlaciones pueden incrementarse en un cierto grupo de deudores, que por otro lado parecen no estar relacionados. Por ejemplo, un grupo de créditos hipotecarios a empleados de una gran compañía que cae en bancarrota. El modelo facilita la detección de este tipo de concentraciones dado que puede manejar segmentaciones arbitrarias de la cartera, y es relativamente simple examinar segmentos particulares de la cartera bajo condiciones extremas de probabilidad de incumplimiento y correlación y analizar sus consecuencias.

El comportamiento aleatorio de las probabilidades de impago introduce otros tipos de dificultades técnicas. Obviamente no existe razón para que las probabilidades de impago no dependan de factores de riesgo y de elementos crediticios, a través de modelos logit como en Credit Portfolio View, o como combinaciones lineales de estos factores como proponen Krenin y Rosen. Los resultados obtenidos pueden ser incluidos en modelos de simulación que generan escenarios de trayectorias para estas variables en el tiempo, y que muestran las típicas discontinuidades de los eventos relacionados de impago. Con los escenarios generados, se puede obtener la distribución de la razón adecuada de capitalización que debe mantenerse para conservar a la concentración bajo control, y/o la distribución de la cota que establece un límite a la concentración que tenga una probabilidad alta de satisfacerse. De hecho, este tipo de experimento puede ser precisamente lo que se necesita para establecer las normas apropiadas al cociente de capitalización y el nivel adecuado de la concentración. Nótese que dado que el proceso de simulación es en este caso para sólo algunas variables, puede hacerse de forma eficiente. Además, es posible que bajo supuestos semejantes a los que hace CreditRisk ${ }^{+}$sobre la distribución de los factores de riesgo, se pueda obtener una solución cerrada para la distribución del VaR, manteniendo la elegancia del modelo. 
Cualquiera que sea la dinámica de las variables, siempre es posible hacer los ajustes necesarios en el tiempo, rastreando sólo algunas variables. Todas estas consideraciones puntualizan nuevas direcciones para las investigaciones futuras, que pueden enriquecer considerablemente los resultados hasta ahora obtenidos. 


\section{Apéndice A}

\section{Teorema 6.1.}

Si se requiere que $\mathrm{F} \leq \theta \mathrm{V} 1$, entonces $\mathrm{H}(\mathrm{F}) \leq \theta$ y la máxima concentración bajo la medida de Herfindahl-Hirschman ocurre si y sólo si F es alguna permutación de la distribución siguiente:

$$
\begin{aligned}
& f_{k}^{*}=\left\{\begin{array}{c}
\theta V ; k=0,1,2,3, \ldots, n \\
0 ; k=n+1, n+2, \ldots, N
\end{array}\right. \\
& \text { donde } n \theta V=V \Rightarrow n \theta=1 o n=1 / \theta
\end{aligned}
$$

\section{Prueba:}

Sólo se probará la cuestión de la optimización, dado que la primera parte se probó en el texto. Para probar necesidad, nótese que la máxima concentración bajo el límite individual en el sentido de la definición 1, se puede obtener resolviendo el problema siguiente de optimización:

$$
\begin{aligned}
\max \mathbf{F}^{\mathrm{T}} \mathbf{F} & \\
\text { s.a. : } \mathbf{1}^{\mathrm{T}} \mathbf{F} & =\mathrm{V} \\
\mathbf{F} & \leq \theta \mathrm{V} \mathbf{1} \\
\mathbf{F} & \geq 0
\end{aligned}
$$

Haciendo $\mathrm{F}=\mathrm{aV}$, donde "a" es tal que $1 \mathrm{Ta}=1$, entonces el problema anterior es equivalente al siguiente:

$$
\left\{\begin{array}{r}
\max \mathbf{a}^{\mathbf{T}} \mathbf{a} \\
\text { s.a }: \mathbf{1}^{\mathbf{T}} \mathbf{a}=1 \\
\theta \mathbf{1}-\mathbf{a} \geq 0 \\
\mathbf{a} \geq 0
\end{array}\right.
$$

Para examinar las condiciones de Karush-Kuhn-Tucker (KKT), defínase la función de Lagrange:

$$
L(\mathbf{a}, \lambda, \mathbf{u}, \mathbf{v})=\mathbf{a}^{\mathrm{T}} \mathbf{a}+\lambda\left(1-\mathbf{1}^{\mathrm{T}} \mathbf{a}\right)+\mathbf{v}^{\mathrm{T}}(\theta \mathbf{1}-\mathbf{a})+\mathbf{u}^{\mathrm{T}} \mathbf{a}
$$

De la cual, las condiciones de optimalidad de primer orden conllevan a:

$$
\nabla_{\mathrm{a}} L=2 \mathbf{a}-\lambda \mathbf{1}-\mathbf{v}+\mathbf{u}=\mathbf{0} \Rightarrow \mathbf{a}^{*}=\frac{1}{2}(\lambda \mathbf{1}+\mathbf{v}-\mathbf{u}) \ldots(6.1)
$$


Nótese que para cualquier vector " $\mathrm{x}$ " tal que $\mathrm{xTx}=\theta$, entonces para cualquier vector " $\mathrm{y}$ " que sea simplemente una permutación de los elementos de "x", también se cumple que yTy= $\theta$. Entonces, si $n \theta=1$, sin perdida de generalidad se mostrará que cualquier permutación de:

$$
a_{i}=\left\{\begin{array}{lll}
\theta & \text { si } & i=1, \ldots, n \\
0 & \text { si } & i=n+1, \ldots, N
\end{array}\right.
$$

es una solución óptima al problema P.1.

Claramente la solución es viable ya que todos los componentes son no negativos y suman uno. Además, ninguno de los elementos es mayor a $\theta$. Por lo tanto, sólo es necesario encontrar los valores de los multiplicadores $\lambda$, u y v consistentes con la solución propuesta y que satisfagan las condiciones complementarias; esto es:

$$
\left.\begin{array}{l}
u_{i} a_{i}=0 \\
v_{i}\left(\theta-a_{i}\right)=0
\end{array}\right\} \forall i
$$

Sea, $u_{i}=\theta-a_{i}$ and $v_{i}=a_{i}$, y la complementariedad se verifica fácilmente.

Para probar suficiencia, se introducen variables de holgura "h", y el problema se reescribe de la manera siguiente:

$$
\left.\begin{array}{ll}
\max & \mathbf{a}^{\mathbf{T}} \mathbf{a} \\
\text { s.a. } & \mathbf{1}^{\mathbf{T}} \mathbf{a}=1 \\
& \mathbf{I} \mathbf{a}+\mathbf{I h}=\theta \mathbf{1} \\
\mathbf{a} \geq \mathbf{0}, \mathbf{h} \geq \mathbf{0}
\end{array}\right\} \quad \ldots . .\left(\mathrm{P}^{\prime} .1 .\right)
$$

Como se trata de maximizar una función convexa en un poliedro definido por un conjunto de restricciones lineales, la solución óptima del problema se encuentra en un punto extremo del poliedro convexo31. Los puntos extremos del poliedro están asociados a las bases de la matriz de restricciones. En lo que sigue, se muestra que estas bases y sus inversas pueden caracterizarse de manera que todos los puntos extremos óptimos del poliedro pueden ser identificados y son de

\footnotetext{
${ }^{31}$ Véase por ejemplo Márquez (1987) o Nash y Sofer (1996)
} 
hecho, permutaciones de la solución propuesta. Reescribiendo las restricciones de manera matricial, se obtiene:

$$
\begin{aligned}
& \mathbf{A}\left[\begin{array}{l}
\mathbf{a} \\
\mathbf{h}
\end{array}\right]=\mathbf{b} \\
& \mathbf{a} \geq \mathbf{0} ; \quad \mathbf{h} \geq \mathbf{0}
\end{aligned}
$$

Donde:

$$
\mathbf{A}=\left[\begin{array}{ccc}
\mathbf{1}_{n}^{\mathbf{T}} & \vdots & \mathbf{0}_{n}^{\mathbf{T}} \\
\cdots & \cdots & \cdots \\
\mathbf{I}_{N} & \vdots & \mathbf{I}_{N}
\end{array}\right] ; \quad \mathbf{a}=\left[\begin{array}{c}
a_{1} \\
\vdots \\
a_{N}
\end{array}\right] \quad \mathbf{h}=\left[\begin{array}{c}
h_{1} \\
\vdots \\
h_{N}
\end{array}\right] \quad \mathbf{b}=\left[\begin{array}{c}
1 \\
\theta \\
\vdots \\
\theta
\end{array}\right]
$$

Es fácil verificar que cada solución básica está asociada a una base compuesta de $\mathrm{N}+1$ columnas de A linealmente independientes, y tienen necesariamente la forma:

$$
\mathbf{B}=\left[\begin{array}{ccccc}
\mathbf{1}_{n}^{\mathbf{T}} & \vdots & \mathbf{0}_{N-n}^{\mathbf{T}} & \vdots & 0 \\
\cdots & \cdots & \cdots & \ldots & \ldots \\
& & & \vdots & \\
& \mathbf{I}_{N} & & \vdots & \mathbf{e}_{1} \\
& & & \vdots &
\end{array}\right]
$$

$\mathbf{1}_{\mathrm{n}}$ es un vector suma de $n$ componentes, $0_{\mathrm{N}-\mathrm{n}}$ es una matriz de $(N-n \times N-n)$ de ceros, $\mathrm{e}_{\mathrm{i}}$ es un vector unidad donde el iésimo elemento es uno y los demás elementos son zero. Nótese que no se pierde generalidad suponiendo que es siempre $\mathrm{e}_{1}$. También puede verificarse que la inversa del anterior tiene la forma:

$$
\mathbf{B}^{-1}=\left[\begin{array}{ccccccc}
1 & \vdots & 0 & \vdots & -\mathbf{1}_{n-1}^{\mathbf{T}} & \vdots & \mathbf{0}_{N-n}^{\mathbf{T}} \\
\cdots & \vdots & \cdots & \vdots & \cdots & \vdots & \cdots \\
& \vdots & & \vdots & \mathbf{I}_{n-1} & \vdots & \mathbf{0}_{n-1 \times N-n} \\
\mathbf{0}_{N-n} & \vdots & \mathbf{0}_{N-n} & \vdots & \cdots & \vdots & \cdots \\
& \vdots & & \vdots & \mathbf{0}_{N-n \times n-1} & \vdots & \mathbf{I}_{N-n} \\
\cdots & \vdots & \cdots & \vdots & \cdots & \vdots & \cdots \\
-1 & \vdots & 1 & \vdots & \mathbf{1}_{n-1}^{\mathbf{T}} & \vdots & \mathbf{0}_{N-n}^{\mathbf{T}}
\end{array}\right]
$$


Multiplicando la inversa por el vector de términos independientes se obtiene:

$$
\mathbf{B}^{-1} \mathbf{b}=\left[\begin{array}{ccccccc}
1 & \vdots & 0 & \vdots & -\mathbf{1}_{n-1}^{\mathbf{T}} & \vdots & \mathbf{0}_{N-n}^{\mathbf{T}} \\
\cdots & \vdots & \cdots & \vdots & \cdots & \vdots & \cdots \\
& \vdots & & \vdots & \mathbf{I}_{n-1} & \vdots & \mathbf{0}_{n-1 \times N-n} \\
\mathbf{0}_{N-n} & \vdots & \mathbf{0}_{N-n} & \vdots & \cdots & \vdots & \cdots \\
& \vdots & & \vdots & \mathbf{0}_{N-n \times n-1} & \vdots & \mathbf{I}_{N-n} \\
\cdots & \vdots & \cdots & \vdots & \cdots & \vdots & \cdots \\
-1 & \vdots & 1 & \vdots & \mathbf{1}_{n-1}^{\mathbf{T}} & \vdots & \mathbf{0}_{N-n}^{\mathbf{T}}
\end{array}\right]\left[\begin{array}{c}
1 \\
\theta \\
\theta \\
\vdots \\
\theta \\
0
\end{array}\right]=\left[\begin{array}{c}
\theta \\
\theta \\
\\
\vdots \\
\\
\\
\\
\\
\end{array}\right.
$$

Moviendo filas y columnas como convenga, se observa que $\mathbf{B}^{-1} \mathbf{b}=\left[\begin{array}{c}\theta \mathbf{1}_{\mathbf{N}} \\ 0\end{array}\right]$, para cualquier base $\mathrm{B}$ de A. Como habría de probarse, esto significa que todos los puntos extremos del poliedro son alguna permutación de:

$$
a_{i}^{n}=\left\{\begin{array}{lll}
\theta & \text { si } & i=1, \ldots, n \\
0 & \text { si } & i=n+1, \ldots, N
\end{array}\right.
$$

\section{Teorema 6.2.}

Si $\mathrm{H}(\mathbf{F}) \leq \theta$ entonces:

$$
f_{i} \leq \frac{1}{N}(1+\sqrt{(N \theta-1)(N-1)}) V<\sqrt{\theta} V \quad \text { para } \mathrm{i}=1,2,3, \ldots, \mathrm{N}
$$

\section{Prueba:}

La idea detrás de la demostración es que sólo se puede tener un crédito muy grande respetando la restricción. Como en la prueba del teorema anterior, redefiniendo $\mathrm{F}$ en términos de las proporciones del valor total de la cartera "a" significa que el crédito máximo bajo las restricciones como una proporción del valor de la cartera, se obtiene resolviendo el siguiente problema de optimización:

$$
\begin{array}{r}
\max \mathbf{e}_{\mathbf{i}}^{\mathbf{T}} \mathbf{a} \\
\text { s.a. } \mathbf{a}^{\mathbf{T}} \mathbf{a}=\theta \\
\mathbf{1}^{\mathrm{T}} \mathbf{a}=1 \\
\mathbf{a} \geq \mathbf{0}
\end{array}
$$


Una vez más, las condiciones necesarias de primer orden se examinan con el Lagrangeano:

$$
L(\mathbf{a}, \lambda, \mu, \mathbf{u})=\mathbf{e}_{i}^{T} \mathbf{a}-\lambda\left(\mathbf{a}^{T} \mathbf{a}-\theta\right)-\mu\left(\mathbf{1}^{T} \mathbf{a}-1\right)-\mathbf{u}^{T} \mathbf{a}
$$

Tomando derivadas parciales con respecto a "a", se puede ver que la solución al problema debe tener la siguiente forma:

$$
\begin{aligned}
\nabla_{\mathrm{a}} \mathrm{L} & =\mathbf{e}_{\mathrm{i}}-\mu \mathbf{1}-\mathbf{u}-2 \lambda \mathbf{a}=0 \\
\Rightarrow \quad \mathbf{a}^{*} & =\frac{\mathbf{e}_{\mathrm{i}}-\mu * \mathbf{1}-\mathbf{u}}{2 \lambda * \ldots \ldots \ldots \ldots} . . . . . . .
\end{aligned}
$$

Sucesivamente, tomando el producto interior de $\mathrm{a}^{*} \operatorname{con} 1, \mathrm{e}_{\mathrm{i}}, \mathrm{e}_{\mathrm{k}}$ después de un poco de álgebra se llega a:

$$
\begin{array}{rr} 
& \mathbf{1}^{\mathrm{T}} \mathbf{a}^{*}=\frac{1-\mu * \mathrm{~N}-\mathbf{1}^{\mathrm{T}} \mathbf{u}^{*}}{2 \lambda *}=1 \\
\Rightarrow & 2 \lambda *=1-\mu * \mathrm{~N}-\mathbf{1}^{\mathrm{T}} \mathbf{u}^{*}
\end{array}
$$

(Dado que $\mathbf{1}^{\mathrm{T}} \mathbf{1}=N$ )

$$
\begin{aligned}
(\mathbf{a} *)^{\mathrm{T}} \mathbf{a}^{*} & =\frac{a_{i}^{*}-\mu^{*}-(\mathbf{u} *)^{\mathrm{T}} \mathbf{a}^{*}}{2 \lambda *}=\theta \\
\Rightarrow a_{i}^{*} & =\mu *+2 \theta \lambda * \ldots \ldots \ldots \ldots
\end{aligned}
$$

(Dado que por complementariedad $\left(\mathbf{u}^{*}\right)^{\mathrm{T}} \mathbf{a}^{*}=0 ;$ y $\left.\mathbf{1}^{\mathbf{T}} \mathbf{a}^{*}=1\right)$.

$$
\begin{gathered}
\mathbf{e}_{\mathrm{i}}^{T} \mathbf{a}^{*}=\frac{1-\mu^{*}-u_{i}^{*}}{2 \lambda *}=a_{i} * \\
\mathbf{e}_{\mathrm{k}}^{\mathrm{T}} \mathbf{a}^{*}=\frac{-\mu^{*}-\mathrm{u}_{\mathrm{i}}^{*}}{2 \lambda *}=\mathrm{a}_{\mathrm{k}} \cdots \cdots
\end{gathered}
$$

Finalmente, una inspección a (6.1), (6.2), (6.3), (6.4) y (6.5) indica que todas las soluciones al problema tienen la forma:

$$
a_{i}= \begin{cases}\alpha>0 & \text { si } k=i \\ \beta \neq \alpha ; \beta>0 & \text { si } k=1, \ldots, n \quad k \neq i \quad \ldots . .(6.6) \\ 0 & \text { en otro caso }\end{cases}
$$


Para verificar que la solución propuesta satisfaga las condiciones KKT, primero nótese que por complementariedad $a_{k}>0$ requiere $u_{k}=0$ o $k=1,2,3, \ldots, n$ y por tanto $u_{i}=0$.

Substituyendo $\alpha=a_{i}$ en (6.3) y (6.4) y resolviendo para $\lambda^{*}$ se obtiene:

$$
\begin{aligned}
& \lambda^{*}=\frac{1-\mu^{*}}{2 \alpha} \ldots \ldots \ldots \\
& \lambda^{*}=\frac{\alpha-\mu^{*}}{2 \theta} \ldots \ldots \ldots
\end{aligned}
$$

Igualando (6.6) y (6.7) y resolviendo para $\mu$ :

$$
\mu=\frac{\theta-\alpha^{2}}{\theta-\alpha}
$$

Del mismo modo, la solución propuesta junto con los resultados de complementariedad en $u_{k}>0$ $(k=n+1, \ldots, N)$, y $u_{k}>0 \Rightarrow a_{k}=0$. Así, de (6.5) se obtiene:

$$
u_{k}=-\mu
$$

Dado que $\mathbf{1}^{\mathrm{T}} \mathrm{u}=(N-n) \mu$, la siguiente ecuación junto con (6.2) conduce a:

$$
2 \lambda=1-n \mu
$$

Ahora, sustituyendo $\mu$ en (6.6):

$$
\begin{aligned}
& 2 \lambda=\frac{1}{\alpha}(1-\mu) \quad \Rightarrow \\
& 2 \lambda=\frac{\alpha-1}{\theta-\alpha} \ldots \ldots \ldots \ldots \ldots \ldots \ldots \ldots . . .
\end{aligned}
$$

Igualando (6.8) y (6.9) y sustituyendo $\mu$ :

$$
\frac{\alpha-1}{\theta-\alpha}=1-n\left(\frac{\theta-\alpha^{2}}{\theta-\alpha}\right) \Rightarrow n \alpha^{2}-2 \alpha-\theta(n-1)+1=0
$$

Resolviendo la ecuación cuadrática: 


$$
\alpha=\frac{1}{n}[1+\sqrt{(n \theta-1)(n-1)}]
$$

Donde la única raíz relevante es la positiva. Dado que $\beta=\frac{1-\alpha}{n-1}$, entonces:

$$
\beta=\frac{1}{n-1}-\frac{1}{n(n-1)}[1+\sqrt{(n \theta-1)(n-1)}]
$$

Es importante notar que estos valores son significativos sólo cuando $\theta \geq 1 / n$. También es interesante notar qué ocurre bajo los valores extremos de $\theta$ : Si $\theta=1$ entonces $\alpha=1, \beta=0$ y si $\theta=1 / n$, entonces $\alpha=1 / n=\beta$.

Ahora debe mostrarse que los valores de $\alpha$ y $\beta$ están asociados a los multiplicadores de Lagrange $\lambda^{*}, \mu^{*} y u^{*}$ que satisfacen las condiciones KKT. De (6.4) y (6.5), y debido a la complementariedad, se observa que:

$$
a_{i}^{*}=\frac{1-\mu^{*}}{2 \lambda *}, \text { y } a_{j}=-\frac{\mu^{*}}{2 \lambda *}(1 \leq \mathrm{i} \leq \mathrm{n}, \mathrm{i} \neq \mathrm{j})
$$

De manera similar:

$$
\mathrm{u}_{\mathrm{k}}^{*}=-\mu^{*}(\mathrm{k}=\mathrm{n}+1, \ldots, \mathrm{N})
$$

De (6.9) es claro que las condiciones KKT se satisfacen si y solo si:

$$
\mu^{*}<0, \lambda^{*} \geq 0 \text { y } 2 \lambda^{*} \geq-\mu^{*}
$$

Por tanto, se debe mostrar que $\mu^{*<0}$, por (6.9) es lo mismo que probar que:

$$
\alpha^{2} \leq \theta<\alpha
$$

Obviamente, $\alpha^{2}<\theta$ y para probar que $\theta<\alpha$ nótese que:

$$
\alpha=\frac{1}{n}(1+\sqrt{(n \theta-1)(n-1)})>\theta \quad \Leftrightarrow \sqrt{(n \theta-1)(n-1)}>n \theta-1 \Leftrightarrow n-1>n \theta-1
$$

que es siempre cierto cuando $\theta<1$. 
Para concluir la prueba, dado que el problema es no convexo, el máximo está en uno de los extremos, entonces debe determinarse el valor de "n" donde se obtiene el máximo. Esto es simple ya que es fácil ver que $\alpha$ es una función monótona creciente de “ $n$ ”, si $\theta<1$, porque en este caso $\frac{d \alpha}{d n}>0$, así que:

$$
\alpha *(N)=\frac{1}{N}(1+\sqrt{(N \theta-1)(N-1)})
$$

Finalmente nótese que:

$$
\alpha *(N)<\sqrt{\theta}=\lim _{N \rightarrow \infty} a *(N)
$$

lo cual concluye la prueba. 
APÉNDICE B

\section{EJEMPLO 7.1}

Segmentación de la matriz de covarianzas M:

\section{Cuadro B.2: $\mathrm{M}_{1}$}

$\begin{array}{lllllllll} & \mathbf{1} & \mathbf{2} & \mathbf{3} & \mathbf{4} & \mathbf{5} & \mathbf{6} & \mathbf{7} & \mathbf{8} \\ \mathbf{1} & 0.0162 & 0.0050 & 0.0050 & 0.0060 & 0.0060 & 0.0082 & 0.0082 & 0.0105 \\ \mathbf{2} & 0.0050 & 0.0475 & 0.0086 & 0.0103 & 0.0103 & 0.0140 & 0.0140 & 0.0180 \\ \mathbf{3} & 0.0050 & 0.0086 & 0.0475 & 0.0103 & 0.0103 & 0.0140 & 0.0140 & 0.0180 \\ \mathbf{4} & 0.0060 & 0.0103 & 0.0103 & 0.0694 & 0.0125 & 0.0169 & 0.0169 & 0.0217 \\ \mathbf{5} & 0.0060 & 0.0103 & 0.0103 & 0.0125 & 0.0694 & 0.0169 & 0.0169 & 0.0217 \\ \mathbf{6} & 0.0082 & 0.0140 & 0.0140 & 0.0169 & 0.0169 & 0.1275 & 0.0230 & 0.0295 \\ \mathbf{7} & 0.0082 & 0.0140 & 0.0140 & 0.0169 & 0.0169 & 0.0230 & 0.1275 & 0.0295 \\ \mathbf{8} & 0.0105 & 0.0180 & 0.0180 & 0.0217 & 0.0217 & 0.0295 & 0.0295 & 0.2100\end{array}$

Cuadro B.3: $\mathrm{C}_{12}{ }^{\mathrm{T}}=\mathrm{C}_{21}$

$\begin{array}{ccccccccc} & \mathbf{1} & \mathbf{2} & \mathbf{3} & \mathbf{4} & \mathbf{5} & \mathbf{6} & \mathbf{7} & \mathbf{8} \\ \mathbf{9} & 0.0063 & 0.0108 & 0.0108 & 0.0130 & 0.0130 & 0.0177 & 0.0177 & 0.0227 \\ \mathbf{1 0} & 0.0081 & 0.0138 & 0.0138 & 0.0166 & 0.0166 & 0.0226 & 0.0226 & 0.0290 \\ \mathbf{1 1} & 0.0081 & 0.0138 & 0.0138 & 0.0166 & 0.0166 & 0.0226 & 0.0226 & 0.0290 \\ \mathbf{1 2} & 0.0111 & 0.0190 & 0.0190 & 0.0229 & 0.0229 & 0.0311 & 0.0311 & 0.0399 \\ \mathbf{1 3} & 0.0111 & 0.0190 & 0.0190 & 0.0229 & 0.0229 & 0.0311 & 0.0311 & 0.0399 \\ \mathbf{1 4} & 0.0132 & 0.0226 & 0.0226 & 0.0273 & 0.0273 & 0.0370 & 0.0370 & 0.0475 \\ \mathbf{1 5} & 0.0169 & 0.0290 & 0.0290 & 0.0350 & 0.0350 & 0.0475 & 0.0475 & 0.0609 \\ \mathbf{1 6} & \mathbf{0 . 0 1 6 9} & 0.0290 & 0.0290 & 0.0350 & 0.0350 & 0.0475 & 0.0475 & 0.0609\end{array}$

Cuadro B.4: $\mathrm{C}_{13}{ }^{\mathrm{T}}=\mathrm{C}_{31}$

$\begin{array}{lllllllll} & \mathbf{1} & \mathbf{2} & \mathbf{3} & \mathbf{4} & \mathbf{5} & \mathbf{6} & \mathbf{7} & \mathbf{8} \\ \mathbf{1 7} & 0.0039 & 0.0067 & 0.0067 & 0.0081 & 0.0081 & 0.0109 & 0.0109 & 0.0140 \\ \mathbf{1 8} & 0.0052 & 0.0089 & 0.0089 & 0.0108 & 0.0108 & 0.0146 & 0.0146 & 0.0188 \\ \mathbf{1 9} & 0.0067 & 0.0114 & 0.0114 & 0.0138 & 0.0138 & 0.0187 & 0.0187 & 0.0240 \\ \mathbf{2 0} & 0.0081 & 0.0138 & 0.0138 & 0.0167 & 0.0167 & 0.0226 & 0.0226 & 0.0290 \\ \mathbf{2 1} & 0.0092 & 0.0157 & 0.0157 & 0.0190 & 0.0190 & 0.0257 & 0.0257 & 0.0330 \\ \mathbf{2 2} & 0.0109 & 0.0187 & 0.0187 & 0.0226 & 0.0226 & 0.0306 & 0.0306 & 0.0393 \\ \mathbf{2 3} & 0.0140 & 0.0240 & 0.0240 & 0.0290 & 0.0290 & 0.0393 & 0.0393 & 0.0504 \\ \mathbf{2 4} & 0.0140 & 0.0240 & 0.0240 & 0.0290 & 0.0290 & 0.0393 & 0.0393 & 0.0504 \\ \mathbf{2 5} & 0.0140 & 0.0240 & 0.0240 & 0.0290 & 0.0290 & 0.0393 & 0.0393 & 0.0504\end{array}$


Cuadro B.5: $\mathrm{M}_{2}$

$\begin{array}{ccccccccc} & \mathbf{9} & \mathbf{1 0} & \mathbf{1 1} & \mathbf{1 2} & \mathbf{1 3} & \mathbf{1 4} & \mathbf{1 5} & \mathbf{1 6} \\ \mathbf{9} & 0.0291 & 0.0086 & 0.0086 & 0.0118 & 0.0118 & 0.0140 & 0.0180 & 0.0180 \\ \mathbf{1 0} & 0.0086 & 0.0475 & 0.0109 & 0.0150 & 0.0150 & 0.0179 & 0.0230 & 0.0230 \\ \mathbf{1 1} & 0.0086 & 0.0109 & 0.0475 & 0.0150 & 0.0150 & 0.0179 & 0.0230 & 0.0230 \\ \mathbf{1 2} & 0.0118 & 0.0150 & 0.0150 & 0.0900 & 0.0207 & 0.0246 & 0.0316 & 0.0316 \\ \mathbf{1 3} & 0.0118 & 0.0150 & 0.0150 & 0.0207 & 0.0900 & 0.0246 & 0.0316 & 0.0316 \\ \mathbf{1 4} & 0.0140 & 0.0179 & 0.0179 & 0.0246 & 0.0246 & 0.1275 & 0.0376 & 0.0376 \\ \mathbf{1 5} & 0.0180 & 0.0230 & 0.0230 & 0.0316 & 0.0316 & 0.0376 & 0.2100 & 0.0483 \\ \mathbf{1 6} & 0.0180 & 0.0230 & 0.0230 & 0.0316 & 0.0316 & 0.0376 & 0.0483 & 0.2100\end{array}$

$$
\text { Cuadro B.6: } \mathrm{C}_{32}{ }^{\mathrm{T}}=\mathrm{C}_{23}
$$

$\begin{array}{clllllllll} & \mathbf{1 7} & \mathbf{1 8} & \mathbf{1 9} & \mathbf{2 0} & \mathbf{2 1} & \mathbf{2 2} & \mathbf{2 3} & \mathbf{2 4} & \mathbf{2 5} \\ \mathbf{9} & 0.0070 & 0.0093 & 0.0119 & 0.0144 & 0.0164 & 0.0195 & 0.0250 & 0.0250 & 0.0250 \\ \mathbf{1 0} & 0.0089 & 0.0119 & 0.0152 & 0.0184 & 0.0209 & 0.0249 & 0.0320 & 0.0320 & 0.0320 \\ \mathbf{1 1} & 0.0089 & 0.0119 & 0.0152 & 0.0184 & 0.0209 & 0.0249 & 0.0320 & 0.0320 & 0.0320 \\ \mathbf{1 2} & 0.0122 & 0.0164 & 0.0209 & 0.0253 & 0.0288 & 0.0343 & 0.0440 & 0.0440 & 0.0440 \\ \mathbf{1 3} & 0.0122 & 0.0164 & 0.0209 & 0.0253 & 0.0288 & 0.0343 & 0.0440 & 0.0440 & 0.0440 \\ \mathbf{1 4} & 0.0146 & 0.0195 & 0.0249 & 0.0301 & 0.0343 & 0.0408 & 0.0524 & 0.0524 & 0.0524 \\ \mathbf{1 5} & 0.0187 & 0.0250 & 0.0320 & 0.0386 & 0.0440 & 0.0524 & 0.0672 & 0.0672 & 0.0672 \\ \mathbf{1 6} & 0.0187 & 0.0250 & 0.0320 & 0.0386 & 0.0440 & 0.0524 & 0.0672 & 0.0672 & 0.0672\end{array}$

Cuadro B.7: $\mathbf{M}_{3}$

$\begin{array}{llllllllll} & \mathbf{1 7} & \mathbf{1 8} & \mathbf{1 9} & \mathbf{2 0} & \mathbf{2 1} & \mathbf{2 2} & \mathbf{2 3} & \mathbf{2 4} & \mathbf{2 5} \\ \mathbf{1 7} & 0.0162 & 0.0093 & 0.0119 & 0.0144 & 0.0164 & 0.0196 & 0.0251 & 0.0251 & 0.0251 \\ \mathbf{1 8} & 0.0093 & 0.0291 & 0.0160 & 0.0193 & 0.0220 & 0.0262 & 0.0336 & 0.0336 & 0.0336 \\ \mathbf{1 9} & 0.0119 & 0.0160 & 0.0475 & 0.0247 & 0.0281 & 0.0335 & 0.0429 & 0.0429 & 0.0429 \\ \mathbf{2 0} & 0.0144 & 0.0193 & 0.0247 & 0.0694 & 0.0340 & 0.0404 & 0.0519 & 0.0519 & 0.0519 \\ \mathbf{2 1} & 0.0164 & 0.0220 & 0.0281 & 0.0340 & 0.0900 & 0.0461 & 0.0591 & 0.0591 & 0.0591 \\ \mathbf{2 2} & 0.0196 & 0.0262 & 0.0335 & 0.0404 & 0.0461 & 0.1275 & 0.0704 & 0.0704 & 0.0704 \\ \mathbf{2 3} & 0.0251 & 0.0336 & 0.0429 & 0.0519 & 0.0591 & 0.0704 & 0.2100 & 0.0903 & 0.0903 \\ \mathbf{2 4} & 0.0251 & 0.0336 & 0.0429 & 0.0519 & 0.0591 & 0.0704 & 0.0903 & 0.2100 & 0.0903 \\ \mathbf{2 5} & 0.0251 & 0.0336 & 0.0429 & 0.0519 & 0.0591 & 0.0704 & 0.0903 & 0.0903 & 0.2100\end{array}$




\section{REFERENCIAS.}

Adelman, M. A. 1969. Comment on the " $H$ " Concentration Measure as a Numbers-Equivalent. Review of Economics and Statistics, Febrero, pp. 99-101.

Altman Edward I., y Anthony Saunders 1998. Credit risk measurement: Development over the last 20 years. Journal of Banking \& Finance 21 (1998) 1721-1742.

Crouhy, Michel, Dan Galai y Robert Mark: A comparative analysis of current credit risk models. Journal of Banking and Finance 24 (2000) pp. 59-117.

Basle Committee on Banking Supervision, 1999. Credit Risk Modeling: Current Practices and Applications.

Bennet, P. 1984. Applying Portfolio Theory to Global Bank Lending. Journal of Banking and Finance.

Caouette, John B., Edward I. Altman y Paul Narayanan 1998. Managing Credit Risk: The next great challenge. Ed. John Wiley \& Sons, Inc.

Coopers and Lybrand. 1993. Growth and Diversification: Are the Benefits Always There? In Mortgage Banking, editado por J. Lederman. Chicago: Probus Publishing Company.

Cowell, Frank A., Measuring Inequality $2^{\text {nd }}$ edition. LSE Handbooks in Economics, Prentice Hall/Harvester Wheatsheaf, 1995.

Credit Risk + : Credit Suisse Financial Products, New York, N.Y. 1996.

CreditMetricsTM: Technical document, Abril 2, 1997.

De Groot, Morris H., Probabilidad y Estadística, Addison-Wesley Iberoamericana, Wilmington Delaware. 1988.

Encaoua, David y Jacquemin, Alexis. 1980. Degree of monopoly, indices of concentration and threat of entry. International Economic Review 21.

Finger, Christopher C.: Sticks and Stones, The RiskMetrics Group. 1998 
Gordy, Michael B. A comparative Anatomy of Credit Risk Models. Journal of Banking and Finance, vol 24, 2000, pp. 119-149.

Kealhofer, S. Portfolio Management of Default Risk. KMV Corporation, 1998.(Revised.)

Kealhofer, S. Modeling Default Risk. KMV Corporation, 1999 (Revised.)

Kelly Jr. W.A. 1981. A Generalized Interpretation of the Herfindahl Index. Southern Economic Journal, 48(1) pp. 50-57.

Lambert, Peter J. 1993. The Distribution and Redistribution of Income, Ed. Manchester University Press.

Márquez Diez-Canedo, Javier 1987. Fundamentos de Teoría de Optimización, Editorial Limusa S.A. de C.V.

Markowitz, H. 1959. Portfolio Selection: Efficient Diversification of Investments. New York: John Wiley \& Sons.

Mason, S. P. 1995. The allocation of Risk. In The Global Financial System. Boston: Harvard Business School Press.

Merton, R. C. On the pricing of corporate debt: The risk structure of interest rates."Journal of Finance 29, pp. 449-470, 1974.

Moody's Investors Service. 1991. Rating Cash Flow Transactions Backed by Corporate Debt. Moody's Special Report. Abril.

Nash, Stephen G. y Ariela Sofer 1996. Linear and Nonlinear Programming, The McGraw-Hill Companies, Inc.

Neckell, Pamela, W. Perraudin and S. Varotto: Stability of Rating Transitions. Journal of Banking and Finance, vol. 24, pp. 203-227, 2000.

Shy, Oz. 1995. Industrial Organization: Theory and Applications. The MIT press.

Sleuwaegen, Leo E., Raymond R. De Bondt, and Wim V. Dehandschuter. The Herfindahl index and concentration ratios revisited. The Antitrust Bulletin/Fall 1989. 
Theil, Henri 1967. Information Theory, Ed. North Holland Publishing Co.

Tirole, Jean. 1995. The Theory of Industrial Organization. M. I. T. Press.

Weinstock, David S. Some little-known properties of the Herfindahl-Hirschman Index: Problems of Translation and Specification. The Antitrust Bulletin/ Winter 1984.

Wilson, Thomas C. Portfolio Credit Risk (I). Risk magazine, 10(9) Septiembre 1997.

Wilson, Thomas C. Portfolio Credit Risk(II). Risk magazine, 10(10) Octubre 1997. 